\title{
Etude du nettoyage d'un stérilisateur de lait U.H.T. Ordre d'utilisation des détergents alcalin et acide et aspects cinétiques
}

\author{
Marie-Noëlle PERLAT, M. LALANDE, G. CORRIEU \\ I.N.R.A., Laboratoire de Génie industriel alimentaire \\ 369, rue Jules-Guesde - 59650 Villeneuve-d'Ascq cedex, France
}

\begin{abstract}
Résumé
Un des principaux inconvénients du procédé de stérilisation U.H.T. indirect du lait dans des échangeurs à plaques est l'encrassement des surfaces d'échange thermique. Trois fois par jour, les stérilisateurs à plaques doivent être nettoyés afin de rendre aux surfaces d'échange leur capacité thermique et leur propreté initiales. Mais compte tenu du coût élevé de ces nettoyages, il est indispensable d'avoir une parfaite connaissance des phénomènes afin de pouvoir les optimiser.

L'objectif de notre travail est premièrement de déterminer les effets des nettoyages alcalins et acides sur les différents constituants chimiques des dépôts formés lors de l'encrassement et deuxièmement d'évaluer les cinétiques d'enlèvement de ces composés. Chaque section du stérilisateur est étudiée séparément selon deux procédés différents. Le premier (S-A) consiste à nettoyer d'abord avec l'hydroxyde de sodium $(2 \%)$ puis avec l'acide nitrique (2\%) alors que pour le second (A-S), les mêmes solutions sont utilisées mais dans l'ordre inverse. Les conditions de nettoyage sont : $90^{\circ} \mathrm{C}$ pour la température et $400 \mathrm{l} / \mathrm{h}$ pour le débit. Les cinétiques de nettoyage sont étudiées par échantillonnage automatique des solutions nettoyantes à la sortie de la section étudiée afin d'analyser les quantités de matières protéiques, minérales et grasses éliminées et leur vitesse d'élimination.

Sur la section de préchauffage, les deux procédures de nettoyage sont tout à fait satisfaisantes (élimination totale de la souillure macroscopique) mais le procédé A-S est plus rapide. Pour la section de chauffage seul le procédé A-S est efficace ; dans l'autre cas, la totalité des protéines n'a pas été éliminée et à l'ouverture de l'appareil, nous constatons que la surface d'échange est encrassée aux points de contact des plaques d'échange. Des hypothèses concernant la structure des dépôts et leur mécanisme d'élimination sont discutées.
\end{abstract}

Mots clés : Nettoyage - Stérilisation U.H.T. du lait - Cinétiques - Détergents alcalin et acide. 


\section{Introduction}

Le procédé de stérilisation du lait à Ultra Haute Température (U.H.T.) par chauffage indirect est de plus en plus fréquemment utilisé dans l'industrie laitière. Son principal intérêt est de permettre d'importantes récupérations d'énergie (plus de $85 \%$ de l'énergie thermique mise en œuvre) alors que les procédés à chauffage direct (c'est-à-dire à contact direct lait-vapeur) plafonnent à environ $60 \%$. L'un des inconvénients majeurs de ce procédé est l'encrassement des surfaces d'échange de chaleur. Il en résulte d'une part un accroissement de la consommation d'énergie résultant de l'augmentation des pertes de charge et la diminution des coefficients de transfert thermique de l'échangeur, et, d'autre part, une diminution sensible de l'efficacité stérilisatrice de l'appareil au cours du traitement. La mise en œuvre de ce procédé de stérilisation U.H.T. suppose donc la réalisation à intervalles réguliers (toutes les 6 à 8 h) d'opérations de nettoyage et de désinfection dont la durée totale est de 1 à $2 \mathrm{~h}$. En fonctionnement continu, ces opérations occupent généralement $4 \mathrm{~h}$ sur $24 \mathrm{~h}$. Leur coût est loin d'être négligeable dans le coût total du procédé et leur parfaite maîtrise est donc nécessaire à une gestion satisfaisante des installations.

Lors des opérations industrielles de nettoyage des stérilisateurs de lait, l'arrêt du traitement thermique est immédiatement suivi d'un rinçage à l'eau appelé pré-rinçage. L'enlèvement des dépôts adhérant aux parois d'échange de chaleur est ensuite effectué au moyen des solutions d'hydroxyde de sodium et d'acide nitrique aussi bien dans le cas du nettoyage final (arrêt de l'installation) que dans celui des nettoyages intermédiaires. Les durées de passage et les températures des solutions détergentes diffèrent selon le type de nettoyage et les usines considérées. On admet généralement que le détergent alcalin provoque l'élimination de la plupart des constituants organiques des dépôts tandis que le détergent acide attaque plutôt leur fraction minérale. L'ordre d'utilisation de ces solutions n'est pas défini de manière rationnelle et varie d'une usine à l'autre.

La composition et parfois la structure et la répartition géométrique des dépôts ont fait l'objet de plusieurs études (LYSTER, 1965; Burton, 1966 ; Ito et al., 1967 ; Thom, 1970 ; Lalande et al., 1984). L'existence de deux types différents de dépôts selon la zone du stérilisateur considérée est généralement admise. Un dépôt blanchâtre, essentiellement protéique, de structure spongieuse adhère aux surfaces d'échange au contact desquelles le lait est préchauffé (d'environ $80^{\circ} \mathrm{C}$ à $120^{\circ} \mathrm{C}$ ). Un dépôt grisâtre, appelé pierre de lait, de composition essentiellement minérale, apparaît dans la zone de chauffage du lait (jusqu'à la température de stérilisation).

Concernant le processus de nettoyage et les mécanismes qu'il implique les études sont beaucoup moins nombreuses. Cette remar- 
que vaut surtout pour les cinétiques d'enlèvement des dépôts, pourtant fondamentales dans l'optique de la gestion optimale des unités industrielles de stérilisation U.H.T. Les revues bibliographiques de Jennings (1963), KulKarni et al. (1975) et CORRIEU (1981) illustrent cette situation et la difficulté de mettre en œuvre des expériences significatives. Nous retiendrons d'une part, les travaux de JenNings (1957, 1959 a, 1959 b, 1963) et Schlussler $(1970,1976)$, d'autre part ceux de Gallot-Lavallée et al. (1982, 1984).

Les premiers auteurs travaillent sur de petits échantillons : quelques millilitres de lait ou d'un produit laitier éventuellement marqué au $\mathrm{P}_{32}$ sont séchés sur des disques en acier inoxydable ou en verre de quelques centimètres carrés avant étude dynamique du nettoyage. Leur critère de fin de nettoyage est la disparition de l'élément marqué ou un test de mouillabilité du support. Ces auteurs s'accordent pour considérer que le phénomène de nettoyage peut, au moins dans certaines parties de sa courbe d'évolution en fonction du temps, être décrit par une réaction d'ordre un, c'est-à-dire que la vitesse d'élimination, proportionnelle à la quantité de dépôt adhérant à la surface à nettoyer, décroît au fur et à mesure que cette dernière diminue. Schlussler (1976) essaye de montrer que la vitesse de nettoyage est limitée par la diffusion dans la solution détergente du complexe formé par réaction de cette dernière avec le dépôt.

Gallot-Lavallée et al. (1982, 1984) s'intéressent aux dépots résultant de la pasteurisation de 1 à $2,5 \mathrm{~m}^{3}$ de lait entier à l'échelle pilote. Les conditions de vitesse de passage, de turbulence et de température de pasteurisation utilisées permettent d'affirmer que les dépôts étudiés sont tout à fait comparables à ceux obtenus dans les pasteurisateurs industriels. Des portions de tuyauterie en acier inoxydable, d'une surface unitaire d'environ $1,25 \quad 10^{-2} \mathrm{~m}^{2}$, placées entre la sortie de la section de chauffage et l'entrée de la section de chambrage du pasteurisateur à plaques servent de support au dépôt. Ce dernier présente une répartition uniforme. Un capteur optique installé sur le circuit de nettoyage immédiatement après la portion de tuyauterie encrassée permet de mesurer en continu la vitesse d'élimination du dépôt. Avec cette procédure, les auteurs mettent en évidence que la vitesse de nettoyage, nulle au temps zéro, augmente jusqu'à une valeur maximale avant de décroître et de tendre vers zéro. Cette allure a conduit les auteurs à proposer un schéma réactionnel composé de deux réactions en série.

Notre travail aborde les aspects qualitatif et quantitatif du nettoyage d'un stérilisateur de lait U.H.T. à plaques. Nous étudions l'effet de chaque solution détergente sur les dépôts en fonction de la nature et de la composition de ces derniers afin de déterminer l'ordre de passage des solutions qui assure la meilleure efficacité de nettoyage. Les aspects cinétiques du phénomène d'élimination des dépôts sont également abordés. 


\section{Matériels et méthodes}

\section{A. Stérilisateur U.H.T. et traitement du lait}

Deux échangeurs à plaques (Vicarb, modèle V7) sont utilisés lors des traitements thermiques du lait (fig. 1). Les débits de lait choisis pour réaliser la pré-pasteurisation et la stérilisation ainsi que les débits de solutions de nettoyage assurent des conditions de turbu-

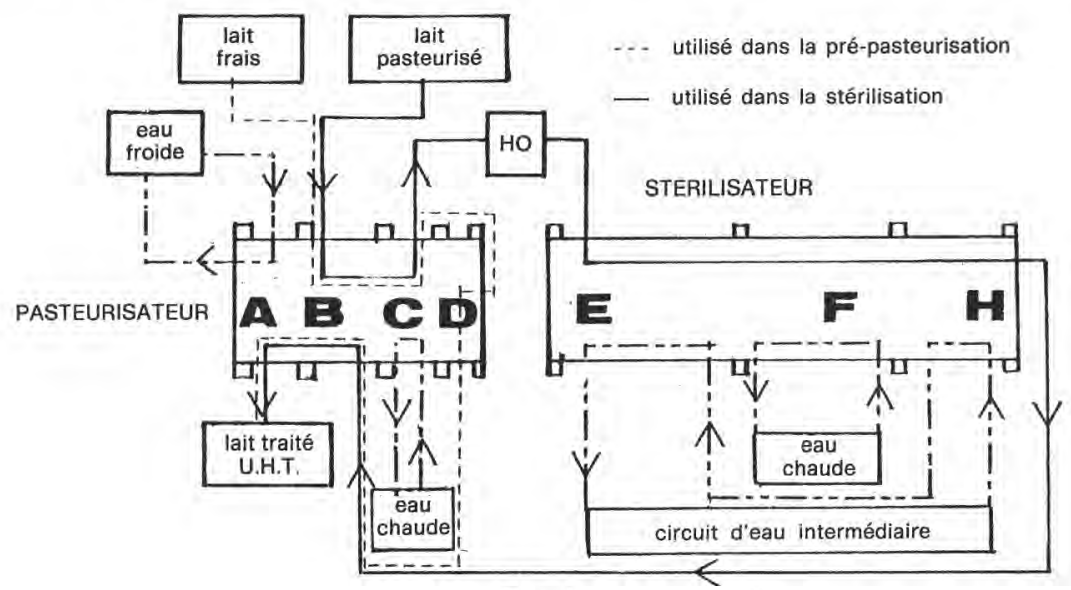

FIG. 1

Schéma de la plate-forme pilote de stérilisation du lait à Ultra Haute Température

$A$, section de refroidissement ; $B$, section de récupération d'énergie ; $C$, section de chauffage; $D$, section de chambrage (utilisée seulement pour la pré-pasteurisation) ; E, section de préchauffage du stérilisateur ; $F$, section de chauffage du stérilisateur ; $H$, section de pré-refroidissement du stérilisateur ; HO, homonéisateur.

Flow diagram of pilot scale plant for sterilization of milk at Ultra-High-Temperature

$A$, cooling section ; $B$, regenerative section ; $C$, heating section ; $D$, holding section (used only in pre-pasteurization) ; E. preheating section of sterilizer ; $F$, heating section of sterilizer ; $H$, precooling section of sterilizer ; $\mathrm{HO}$, homogenizer.

lence tout à fait comparables à celles rencontrées industriellement. En effet, l'appareil pilote utilisé possède des caractéristiques géométriques (largeur des plaques de $0,150 \mathrm{~m}$ et espace entre plaques après serrage de $3,8 \quad 10^{-3} \mathrm{~m}$ ) qui sont homothétiques de celles des appareils industriels. Cette similitude assure des vitesses de circulation dans notre appareil pilote $\left(0,2\right.$ à $\left.0,3 \mathrm{~m} \cdot \mathrm{s}^{-1}\right)$ du même ordre de grandeur que celles couramment employées dans les stérilisateurs industriels. Selon les essais, 2000 à 5000 litres de lait entier de grand mélange, cru, stocké à $4^{\circ} \mathrm{C}$, sont tout d'abord pasteurisés à un 
débit de 400 1/h dans le pasteurisateur qui se compose d'une section de récupération d'énergie (B), d'une section de chauffage (C), d'une section de chambrage (D) et d'une section de refroidissement (A). Le lait ainsi pasteurisé est stocké une journée à $4^{\circ} \mathrm{C}$. Il est ensuite repris, préchauffé dans le pasteurisateur et stérilisé dans le stérilisateur qui se compose d'une section de préchauffage de 20 passes (E), d'une section de chauffage de 16 passes (F) et d'une section de prérefroidissement de 10 passes $(\mathrm{H})$. Les débits de lait et d'eau ssont fixés à $600 \mathrm{l} / \mathrm{h}$ et le lait est homogénéisé (homogénéisateur Manton Gaulin, modèle M3) sous une pression de $18 \mathrm{MPa}$ à $65^{\circ} \mathrm{C}$. L'évolution de la température du lait au sein du pasteurisateur et du stérilisateur, dans les conditions de traitement standard est présentée à la figure 2. Les surfaces des sections de préchauffage (E) et de chauffage (F), respectivement de $3 \mathrm{~m}^{2}$ et de $2,4 \mathrm{~m}^{2}$, s'encrassent lors des traitements thermiques du lait. La surface de la zone de prérefroidissement $(\mathrm{H})$ fixée à $1,5 \mathrm{~m}^{2}$ n'est pas, ou très peu, encrassée.

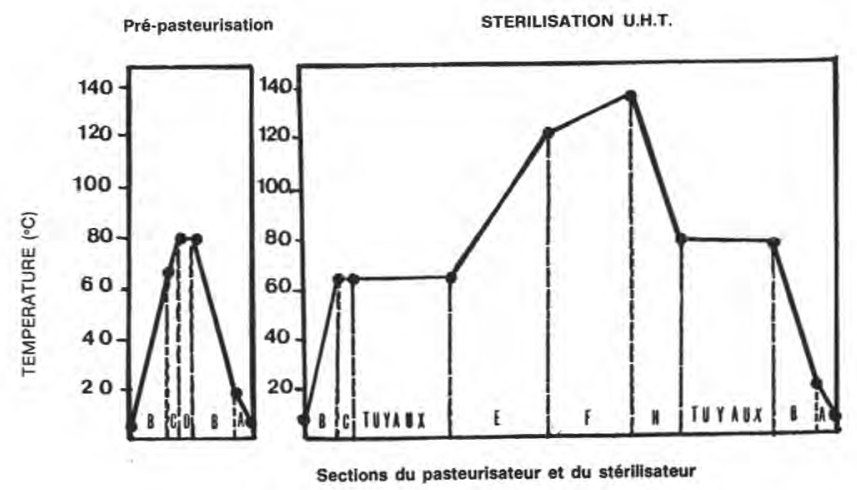

FIG. 2

Evolution de la température du lait au sein des échangeturs à plaques lors de la pré-pasteurisation et de la stérilisation à Ultra Haute Température dans les conditions standard (débit de $400 \mathrm{l} / \mathrm{h}$, homogénéisation à $68^{\circ} \mathrm{C}$, stérilisation à $\left.138^{\circ} \mathrm{C}\right)$.

Temperature gradients used during pre-pasteurization and Ultra-High-Temperature (UHT) sterilization of milk for standard trials (400 $\mathrm{l} / \mathrm{h}$ flow rate; homogenizing at $68^{\circ} \mathrm{C}$; steritization at $138^{\circ} \mathrm{C}$ ).

\section{B. Récupération des dépôts}

A la fin de chaque traitement thermique, le stérilisateur est rincé avec de l'eau douce et démonté. Toutes les plaques sont alors égouttées et immédiatement pesées afin de déterminer leur poids de dépôt frais. L'échangeur étant configuré avec dess passes à un seul 
canal, c'est-à-dire composées de deux plaques, une plaque encrassée sur les deux est séchée à $105^{\circ} \mathrm{C}$ pendant $20 \mathrm{~min}$. Les dépôts secs ainsi obtenus sont recueillis et analysés afin de déterminer leur fraction minérale, protéique et grasse. Les profils de distribution des dépôts sont établis passe par passe. Ils sont exprimés en poids de dépôt sec total et constituant par constituant.

\section{Protocole expérimental du nettoyage}

Chaque section encrassée du stérilisateur est remontée en remplaçant chacune des plaques séchées par une plaque identique propre afin de conserver la même configuration que lors de l'encrassement. De cette façon, le nettoyage concerne une demi-passe encrassée par canal. On peut raisonnablement faire l'hypothèse que ce changement ne modifie pas la cinétique de nettoyage de la plaque encrassée.

Le nettoyage des sections de préchauffage $\mathrm{E}$ et de chauffage $\mathrm{F}$ se fait séparément et en boucle ouverte c'est-à-dire sans recyclage des solutions détergentes. Celles'ci sont d'abord préchauffées dans la section de récupération d'énergie (B) du pasteurisateur ; elles passent ensuite dans l'homogénéisateur et sont chauffées dans la section du stérilisateur à nettoyer ; leur température de sortie est maintenue à $90^{\circ} \mathrm{C}$ à l'aide de la boucle de régulation assurant normalement le contrôle de la température de stérilisation. Les nettoyages alcalin et acide sont respectivement effectués au moyen d'une solution d'hydroxyde de sodium à $2 \%$ et d'une solution d'acide nitrique à $2 \%$ en poids. Un rinçage intermédiaire de $10 \mathrm{~min}$ environ à l'eau douce $(T h=0)$ les sépare. L'ordre de passage de ces solutions, qui varie d'un essai à l'autre, est précisé dans les tableaux 1 et 2 . La notation S-A signifie que la solution d'hydroxyde de sodium est utilisée avant la solution acide. Dans le cas contraire, l'essai est noté A-S. Chaque solution circule au minimum $20 \mathrm{~min}$ dans l'appareil au débit de $400 \mathrm{l} / \mathrm{h}$.

Aucun de nos essais n'a étudié l'effet d'additifs dans les solutions de nettoyage (tensio-actifs ou agents séquestrants) fréquemment utilisés dans l'industrie laitière.

\section{Suivi du nettoyage}

Pour suivre le nettoyage de la zone considérée, le capteur optique utilisé par GALlot-LAvallée et al. $(1982,1984)$ a été abandonné car jugé insuffisamment spécifique. Par contre, des capteuns de température, de débit et de pression différentielle ont été installés sur la plate-forme expérimentale comme indiqué sur la figure 3. Le signal de chaque capteur est acquis sur un micro-calculateur (Leanord, Picolog 80) et converti en mesure physique. 
Analyses des dépôts secs et des solutions de nettoyage prélevés pour la section de préchauffage E d'un stérilisateur U.H.T. à plaques

Analysis of dry deposits and cleaning solutions sampled from the preheating section $E$ of the U.H.T. plate heat sterilizer

\begin{tabular}{|c|c|c|c|c|c|c|c|c|c|c|}
\hline & \multicolumn{4}{|c|}{$\begin{array}{l}\text { Poids secs et pourcentage de chaque } \\
\text { constituant dans les dépôts secs } \\
\text { récupérés sur les plaques }\end{array}$} & \multicolumn{2}{|c|}{$\begin{array}{c}\text { Poids } \\
\text { et pourcentage } \\
\text { des matières } \\
\text { éliminées par le } \\
\text { nettoyage alcalin }\end{array}$} & \multicolumn{2}{|c|}{$\begin{array}{l}\text { Poids } \\
\text { et pourcentage } \\
\text { des matières } \\
\text { éliminées par le } \\
\text { nettoyage acide }\end{array}$} & \multicolumn{2}{|c|}{$\begin{array}{c}\text { Poids totaux } \\
\text { éliminés } \\
\text { par nettoyage } \\
\text { et pourcentage } \\
\text { correspondant par } \\
\text { rapport aux dépôts } \\
\text { initialement } \\
\text { présents }\end{array}$} \\
\hline & $\mathbf{M}_{t}, \mathrm{~g}$ & $\begin{array}{l}P_{d}, g \\
\left(\frac{P_{d}}{M_{t}}, \%\right)\end{array}$ & $\begin{array}{l}M_{d}, g \\
\left(\frac{M_{d}}{M_{t}}, \%\right)\end{array}$ & $\begin{array}{c}\mathrm{G}_{\mathrm{d}}, \mathrm{g} \\
\left(\frac{\mathrm{G}_{\mathrm{d}}}{\mathrm{M}_{\mathrm{t}}}, \%\right)\end{array}$ & $\begin{array}{l}P, g \\
\left(\frac{P}{P_{d}}, \%\right)\end{array}$ & $\begin{array}{l}\mathrm{M}, \mathrm{g} \\
\left(\frac{\mathrm{M}}{\mathrm{M}_{\mathrm{d}}}, \%\right)\end{array}$ & $\begin{array}{c}\mathrm{P}, \mathrm{g} \\
\left(\frac{\mathrm{P}}{\mathrm{P}_{d}}, \%\right)\end{array}$ & $\begin{array}{c}\mathrm{M}, \mathrm{g} \\
\left(\frac{\mathrm{M}}{\mathrm{M}_{\mathrm{d}}}, \%\right)\end{array}$ & $\begin{array}{l}\mathrm{P}, \mathrm{g} \\
\left(\frac{\mathrm{P}}{\mathrm{P}_{\mathrm{d}}}, \%\right)\end{array}$ & $\begin{array}{l}\mathrm{M}, \mathrm{g} \\
\left(\frac{\mathrm{M}}{\mathrm{M}_{\mathrm{d}}}, \%\right)\end{array}$ \\
\hline $\begin{array}{l}\text { Essai } 1 \\
\text { S-A }\end{array}$ & 138,6 & $\begin{array}{l}69,6 \\
(50)\end{array}$ & $\begin{array}{l}57,3 \\
(41)\end{array}$ & $\begin{array}{l}4,1 \\
(2)\end{array}$ & $\begin{array}{l}65,9 \\
(95)\end{array}$ & $\begin{array}{l}43,2 \\
(76)\end{array}$ & - & $\begin{array}{l}11,7 \\
(20)\end{array}$ & $\begin{array}{l}65,9 \\
(95)\end{array}$ & $\begin{array}{l}54,9 \\
(96)\end{array}$ \\
\hline $\begin{array}{l}\text { Essai } 2 \\
\text { S-A }\end{array}$ & 94,0 & $\begin{array}{l}50,1 \\
(53)\end{array}$ & $\begin{array}{l}36,8 \\
(39)\end{array}$ & $\begin{array}{l}1,8 \\
(2)\end{array}$ & $\begin{array}{l}49,8 \\
(99)\end{array}$ & $\begin{array}{l}24,6 \\
(67)\end{array}$ & - & $\begin{array}{l}14,1 \\
(38)\end{array}$ & $\begin{array}{l}49,8 \\
(99)\end{array}$ & $\begin{array}{r}38,7 \\
(105)\end{array}$ \\
\hline $\begin{array}{l}\text { Essai } 3 \\
\text { S-A }\end{array}$ & 56,8 & $\begin{array}{l}29,4 \\
(52)\end{array}$ & $\begin{array}{l}20,0 \\
(35)\end{array}$ & $\begin{array}{l}4,0 \\
(7)\end{array}$ & $\begin{array}{r}33,7 \\
(115)\end{array}$ & $\begin{array}{l}11,5 \\
(58)\end{array}$ & - & $\begin{array}{l}10,3 \\
(51)\end{array}$ & $\begin{array}{r}33,7 \\
(115)\end{array}$ & $\begin{array}{r}21,8 \\
(109)\end{array}$ \\
\hline $\begin{array}{l}\text { Essai } 4 \\
\text { A-S }\end{array}$ & 116,4 & $\begin{array}{l}61,7 \\
(53)\end{array}$ & $\begin{array}{l}44,2 \\
(38)\end{array}$ & $\begin{array}{l}2,1 \\
(2)\end{array}$ & $\begin{array}{r}62,8 \\
(102)\end{array}$ & $\begin{array}{c}0 \\
(0)\end{array}$ & - & $\begin{array}{r}46,5 \\
(105)\end{array}$ & $\begin{array}{r}62,8 \\
(102)\end{array}$ & $\begin{array}{r}46,5 \\
(105)\end{array}$ \\
\hline $\begin{array}{l}\text { Essai } 5 \\
\text { A-S }\end{array}$ & 69,1 & $\begin{array}{l}30,8 \\
(44)\end{array}$ & $\begin{array}{l}29,4 \\
(42)\end{array}$ & $\begin{array}{l}3,5 \\
(5)\end{array}$ & $\begin{array}{r}35,6 \\
(115)\end{array}$ & $\begin{array}{c}0 \\
(0)\end{array}$ & - & $\begin{array}{r}31,6 \\
(108)\end{array}$ & $\begin{array}{r}35,6 \\
(115)\end{array}$ & $\begin{array}{r}31,6 \\
(108)\end{array}$ \\
\hline
\end{tabular}

$P$, protéines ; $M$, minéraux ; $G$, matières grasses ; indice $d$, dépôt sec ; $M_{t}$, masse totale sèche ; S-A, nettoyage alcalin suivi d'un nettoyage acide ; A-S, nettoyage acide suivi d'un nettoyage alcalin.

$P$, protein matter ; $M$, mineral matter; $G$, fat matter; index $d$, dry deposit ; $M_{t}$, total dry matter ; S-A, alkaline cleaning followed by acid cleaning ; A-S, acid cleaning followed by alkaline cleaning. 
TABLEAU 2

Analyses des dépôts secs et des solutions de nettoyage prélevés pour la section de chauffage $F$ d'un stérilisateur U.H.T. à plaques

Analysis of dry deposits and cleaning solutions sampled from the heating section $F$ of the U.H.T. plate heat sterilizer

\begin{tabular}{|c|c|c|c|c|c|c|c|c|c|c|}
\hline & \multicolumn{4}{|c|}{$\begin{array}{l}\text { Poids secs et pourcentage de chaque } \\
\text { constituant dans les dépôts secs } \\
\text { récupérés sur les plaques }\end{array}$} & \multicolumn{2}{|c|}{$\begin{array}{c}\text { Poids } \\
\text { et pourcentage } \\
\text { des matières } \\
\text { eliminées nar le } \\
\text { nettoyage alcalin }\end{array}$} & \multicolumn{2}{|c|}{$\begin{array}{l}\text { Poids } \\
\text { et pourcentage } \\
\text { des matières } \\
\text { éliminées par le } \\
\text { nettoyage acide }\end{array}$} & \multicolumn{2}{|c|}{$\begin{array}{l}\text { Poids totaux } \\
\text { éliminés } \\
\text { par nettoyage } \\
\text { et pourcentage } \\
\text { correspondant } \\
\text { par rapport } \\
\text { aux dépôts } \\
\text { initialement } \\
\text { nrésents }\end{array}$} \\
\hline & $\mathrm{M}_{\mathrm{t}}, \mathrm{g}$ & $\begin{array}{l}\mathrm{P}_{\mathrm{d}}, \mathrm{g} \\
\left(\frac{\mathrm{P}_{\mathrm{d}}}{\mathrm{M}_{\mathrm{t}}}, \%\right)\end{array}$ & $\begin{array}{l}M_{d}, g \\
\left(\frac{M_{d}}{M_{t}}, \%\right)\end{array}$ & $\begin{array}{c}\mathrm{G}_{\mathrm{d}}, \mathrm{g} \\
\left(\frac{\mathrm{G}_{\mathrm{d}}}{\mathrm{M}_{\mathrm{t}}}, \%\right)\end{array}$ & $\begin{array}{l}\mathrm{P}, \mathrm{g} \\
\left(\frac{\mathrm{P}}{\mathrm{P}_{\mathrm{d}}}, \%\right)\end{array}$ & $\begin{array}{l}\mathrm{M}, \mathrm{g} \\
\left(\frac{\mathrm{M}}{\mathrm{M}_{\mathrm{d}}}, \%\right)\end{array}$ & $\begin{array}{l}\stackrel{P}{P}, g \\
\left(\frac{P}{P_{d}}, \%\right)\end{array}$ & $\begin{array}{l}\mathrm{M}, \mathrm{g} \\
\left(\frac{\mathrm{M}}{\mathrm{M}_{\mathrm{d}}}, \%\right)\end{array}$ & $\begin{array}{l}\stackrel{P}{P}, g \\
\left(\frac{P}{P_{d}}, \%\right)\end{array}$ & $\begin{array}{c}\mathrm{M}, \mathrm{g} \\
\left(\frac{\mathrm{M}}{\mathrm{M}_{\mathrm{d}}}, \%\right)\end{array}$ \\
\hline $\begin{array}{l}\text { Essai } 6 \\
\text { S-A }\end{array}$ & 222,8 & $\begin{array}{l}31,3 \\
(14)\end{array}$ & $\begin{array}{c}175,0 \\
(78)\end{array}$ & $\begin{array}{l}3,7 \\
(2)\end{array}$ & $\begin{array}{l}25,0 \\
(80)\end{array}$ & $\begin{array}{l}0,0 \\
(0)\end{array}$ & $\begin{array}{l}1,4 \\
(4)\end{array}$ & $\begin{array}{l}183,0 \\
(105)\end{array}$ & $\begin{array}{l}26,4 \\
(84)\end{array}$ & $\begin{array}{c}183,0 \\
(105)\end{array}$ \\
\hline $\begin{array}{l}\text { Essai } 7 \\
\text { S-A }\end{array}$ & 165,5 & $\begin{array}{l}20,8 \\
(13)\end{array}$ & $\begin{array}{l}127,1 \\
(77)\end{array}$ & $\begin{array}{l}4,6 \\
(3)\end{array}$ & $\begin{array}{l}17,2 \\
(83)\end{array}$ & $\begin{array}{l}0,6 \\
(0)\end{array}$ & $\begin{array}{l}0,9 \\
(4)\end{array}$ & $\begin{array}{r}124,2 \\
(98)\end{array}$ & $\begin{array}{l}18,1 \\
(87)\end{array}$ & $\begin{array}{r}124,8 \\
(98)\end{array}$ \\
\hline $\begin{array}{l}\text { Essai } 8 \\
\text { A-S }\end{array}$ & 94,3 & $\begin{array}{l}13,5 \\
(16)\end{array}$ & $\begin{array}{l}72,6 \\
(77)\end{array}$ & $\begin{array}{l}2,4 \\
(3)\end{array}$ & $\begin{array}{r}4,8 \\
(36)\end{array}$ & $\begin{array}{l}1,3 \\
(2)\end{array}$ & $\begin{array}{l}0,8 \\
(6)\end{array}$ & $\begin{array}{l}65,9 \\
(91)\end{array}$ & $\begin{array}{r}5,6 \\
(42)\end{array}$ & $\begin{array}{l}67,2 \\
(93)\end{array}$ \\
\hline $\begin{array}{l}\text { Essai } 9 \\
\text { A-S }\end{array}$ & 171,7 & $\begin{array}{l}21,0 \\
(12)\end{array}$ & $\begin{array}{l}129,8 \\
(76)\end{array}$ & $\begin{array}{l}5,1 \\
\text { (3) }\end{array}$ & $\begin{array}{r}8,7 \\
(41)\end{array}$ & $\begin{array}{l}0,0 \\
(0)\end{array}$ & $\begin{array}{l}0,8 \\
(4)\end{array}$ & $\begin{array}{l}133,0 \\
(102)\end{array}$ & $\begin{array}{r}9,5 \\
(45)\end{array}$ & $\begin{array}{l}133,0 \\
(102)\end{array}$ \\
\hline $\begin{array}{l}\text { Essai } 10 \\
\text { A-S }\end{array}$ & 170,4 & $\begin{array}{l}21,7 \\
(13)\end{array}$ & $\begin{array}{c}130,9 \\
(77)\end{array}$ & $\begin{array}{l}7,7 \\
(4)\end{array}$ & $\begin{array}{l}13,2 \\
(61)\end{array}$ & $\begin{array}{l}0,8 \\
\text { (1) }\end{array}$ & $\begin{array}{l}0,7 \\
(3)\end{array}$ & $\begin{array}{r}128,8 \\
(98)\end{array}$ & $\begin{array}{l}13,9 \\
(64)\end{array}$ & $\begin{array}{r}129,6 \\
(99)\end{array}$ \\
\hline
\end{tabular}

$P$, protéines, $M$, minéraux ; $G$, lipides ; $M_{t}$, masse totale sèche ; indice $d$, dépôt sec ; S-A, nettoyage alcalin suivi d'un nettoyage acide ; A-S, nettoyage acide suivi d'un nettoyage alcalin.

$P$. protein matter ; $M$. mineral matter ; $G$, fat matter ; index $d$, dry deposit ; $M_{t}$, total dry matter ; S-A, alkaline cleaning followed by acid cleaning ; A-S, acid cleaning followed by alkaline cleaning. 


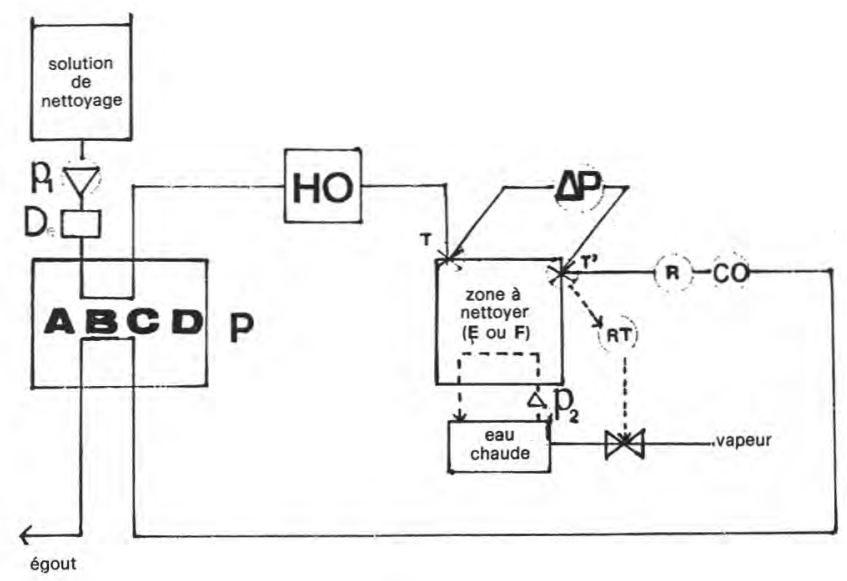

FIG. 3

Représentation schématique de la procédure de nettoyage

$P 1$ et $P 2$, pompes de circulation ; $T$ et $T$, sondes de température : $\Delta P$, capteur de pression différentielle ; $R T$, régulateur de température ; $R$, résistivimètre servant à suivre la concentration de la solution nettoyante ; $\mathrm{CO}$, collecteur d'échantillons ; $H O$, homogénéisateur ; $D_{\theta}$, débitmètre électromagnétique.

Schematic representation of the cleaning procedure

$P 1$ and $P 2$, feed pumps; $T$ and $T^{\prime}$, temperature probes; $\Delta P$, differential pressure sensor ; $R T$, thermoregulator ; $R$, resistivity meter used to follow the concentration of the cleaning solution; $\mathrm{CO}$, sampler ; HO, homogenizer ; $D_{e}$, magnetic flow-meter.

Le capteur de pression différentielle (Schaevitz, type $\mathrm{P}$ 2100) installé entre l'entrée et la sortie de la partie de l'échangeur à nettoyer permet de mesurer l'évolution du taux de colmatage moyen en fonction du temps (CoRrieu et al., 1980 ; LALANDE et al., 1981). Le taux de colmatage (relation 1) traduit directement les variations du diamètre hydraulique équivalent du circuit du fait de la présence de dépôt.

$$
\alpha=\frac{\mathrm{D}_{\mathrm{o}}-\mathrm{D}}{\mathrm{D}_{\mathrm{o}}}
$$

$\mathrm{D}_{0}$ et $\mathrm{D}$ sont respectivement les diamètres hydrauliques équivalents du circuit propre et du circuit encrassé à l'instant de la mesure. $\alpha$ s'exprime également (relation 2 ) en fonction de la perte de charge $\left(\Delta \mathbf{P}_{\circ}\right)$ qu'aurait dans les mêmes conditions de fonctionnement le circuit propre. La valeur de $\Delta \mathrm{P}_{\mathrm{o}}$ est prédite grâce au modèle hydrodynamique du système et à une identification préalable.

$$
\alpha=1-\sqrt[3]{\frac{\Delta \mathrm{P}_{\mathrm{o}}}{\Delta \mathrm{P}}}
$$


Ce principe de mesure permet de suivre en continu l'encrassement et le nettoyage de pasteurisateurs et stérilisateurs à plaques, son application a été brevetée (LALANDE et CORRIEU, 1979).

L'évolution du taux de colmatage nous permet de juger de l'efficacité respective des solutions détergentes utilisées. Lorsqu'il devient nul ou très faible, nous considérons que la quasi-totalité de la souillure macroscopique qui adhérait aux surfaces d'échange a été éliminée.

Dans le cadre de notre travail, nous avons comparé les valeurs du taux de colmatage avant et après nettoyage. Nous noterons respectivement le taux de colmatage initial $\alpha_{i}$ et le taux de colmatage final $\alpha_{\mathrm{e}}$ :

$$
\alpha_{i}=100 \times \frac{\mathrm{D}_{0}-\mathrm{D}_{\mathrm{t}: 0}}{\mathrm{D}_{\mathrm{o}}}=100 \times\left(1-\sqrt[3]{\frac{\Delta \mathrm{P}_{\mathrm{o}}}{\Delta \mathrm{P}_{\mathrm{t}: \mathrm{o}}}}\right)
$$

et

$$
\alpha_{r}=100 \times \frac{\mathrm{D}_{\mathrm{o}}-\mathrm{D}_{\mathrm{t}: \mathrm{te}}}{\mathrm{D}_{\mathrm{s}}}=100 \times\left(1-\sqrt[3]{\frac{\Delta \mathrm{P}_{\mathrm{t}}}{\Delta \mathrm{P}_{\mathrm{t}: \mathrm{tr}}}}\right)
$$

où $\mathrm{D}_{o}$ et $\Delta \mathrm{P}_{0}$ sont respectivement le diamètre hydraulique et la pression différentielle de l'appareil propre.

Parallèlement, un collecteur de fraction prélève des échantillons représentatifs de l'effluent de nettoyage. Les prélèvements sont effectués toutes les 2 secondes pendant la première minute, toutes les 3 secondes pendant la deuxième minute, toutes les 6 secondes pendant la troisième minute et ensuite toutes les 2 min jusqu'à la fin du nettoyage. Une analyse des prélèvements permet de calculer les quantités de matières minérales et protéiques réellement enlevées pendant le nettoyage.

\section{E. Analyses}

- La quantité de protéines totales contenues dans les dépôts et dans les solutions acides de nettoyage est déterminée à partir de la teneur en azote $(\mathrm{N} \times 6,38)$ dosée par la méthode du Kjeldahl (Norme FIL-IDF 20, 1962) avec mesure de la concentration en ammoniaque à l'aide d'une électrode spécifique à ammoniaque (HeLaINE, 1977) : elle a une précision de $5 \%$ environ. La quantité de protéines totales contenue dans les échantillons alcalins est dosée par la méthode du Biuret (Gornall, 1949) : elle a une précision de $5 \%$ environ.

- La quantité de lipides contenues dans les dépôts est déterminée par la méthode décrite par la norme FIL-IDF 1A, 1969. Dans les prélèvements de solutions de nettoyage, les matières grasses sont en trop faible concentration et ne peuvent pas être dosées.

- La fraction minérale contenue dans les dépôts est obtenue par calcination à $550^{\circ} \mathrm{C}$ pendant $16 \mathrm{~h}$. Le calcium est dosé par spec- 
trophotométrie d'absorption atomique et le phosphore par la méthode de Cavell (1955). A partir des quantités de calcium et de phosphore obtenues par analyse, nous déterminons le rapport $\mathrm{Ca} / \mathrm{P}$. Ce qui nous permet de vérifier que ces deux éléments sont essentiellement sous forme de phosphate tricalcique $(\mathrm{Ca} / \mathrm{P}=1,5)$. Etant donné que la fraction minérale des dépôts contient environ $95 \%$ de phosphate tricalcique (LALANDE et al., 1984), la quantité totale de minéraux éliminés par les solutions de nettoyage est obtenue à partir de la teneur en calcium $(\mathrm{Ca} \times 2,7155)$ de façon directe pour les solutions acides et après acidification nitrique pour les solutions alcalines. La précision sur l'analyse du calcium est de $3 \%$.

\section{F. Méthodes de calcul}

L'analyse des solutions de nettoyage permet de calculer la vitesse instantanée d'enlèvement des protéines et des minéraux $\left(\mathrm{V}\left(\mathrm{t}_{\mathrm{I}}\right)\right)$ exprimée en $\mathrm{g} \mathrm{m}^{-2} \mathrm{~s}^{-1}$, par la relation 3 :

$$
\mathrm{V}\left(\mathrm{t}_{\mathrm{r}}\right)=\left(\mathrm{C}\left(\mathrm{t}_{\mathrm{r}}\right) \cdot \mathrm{Q} / \mathrm{S}\right) \cdot 1000
$$

où tr est le temps en seconde auquel l'échantillon I a été prélevé, $Q$ le débit en $\mathrm{m}^{3} / \mathrm{s}, \mathrm{S}$ la surface de la section nettoyée (pour la section de préchauffage $\mathrm{S}=1,275 \mathrm{~m}^{2}$, pour celle de chauffage $\mathrm{S}=$ $1,200 \mathrm{~m}^{2}$ ) et $\mathrm{C}\left(\mathrm{t}_{\mathrm{I}}\right)$ est la concentration en $\mathrm{g} / 1$ de protéines ou de minéraux.

A partir de l'hypothèse que l'association d'un nettoyage alcalin et d'un nettoyage acide permet l'élimination de la totalité des dépôts, il est possible d'évaluer les quantités de protéines et de minéraux adhérant initialement aux plaques encrassées en intégrant en fonction du temps la courbe de vitesse d'enlèvement représentée par la relation 3 .

Ces valeurs peuvent également être déterminées à partir des poids humides en faisant l'hypothèse que la composition et le taux d'humidité des dépôts des deux plaques constituant un même canal sont identiques. Il en résulte une méthode de comparaison des compositions des dépôts secs et des fractions éliminées.

\section{Résultats}

Cinq essais (notés 1 à 5 ) en section de préchauffage $\mathrm{E}$ et cinq essais (notés 6 à 10) en section de chauffage $F$ ont été traités selon. la méthode décrite précédemment. Au cours de ces essais, la quantité de lait utilisée a varié de 1700 litres (essais 5 et 10) à 2600 litres (essais 8). Le débit étant maintenu constant pour tous les essais 
et la qualité des différents laits utilisés étant par ailleurs voisine, les différents essais diffèrent donc essentiellement par leur durée de stérilisation. Les résultats correspondants font l'objet des tableaux 1 et 2 .

\section{A. Composition des dépôts}

Pour nos essais, la composition des dépôts est la suivante :

- la masse totale sèche $\left(\mathrm{M}_{\mathrm{t}}\right)$ représente environ $70 \%$ du poids des dépôts humides adhérant aux plaques encrassées ;

- pour la section de préchauffage E (tabl. 1) la composition moyenne du dépôt sec est de $51 \% \pm 6 \%$ de protéines, de $39 \% \pm$ $4 \%$ de minéraux et de $4 \% \pm 4 \%$ de matières grasses. Pour la section de chauffage $\mathrm{F}$ (tab. 2) la composition moyenne du dépôt sec est de $13,5 \% \pm 3 \%$ de protéines, de $77 \% \pm 2 \%$ de minéraux et de $3 \% \pm 2 \%$ de matières grasses.

\section{B. Quantités de dépôts éliminés par le nettoyage}

Nous avons tout d'abord comparé les quantités totales des matières protéiques et minérales récupérées dans les solutions de nettoyage aux quantités totales qu'il y avait à enlever ; ces quantités sont calculées comme nous l'avons précédemment mentionné. Il est cependant nécessaire de remarquer que seule la précision des méthodes analytiques est connue avec certitude alors qu'en réalité, nous introduisons beaucoup d'autres erreurs, difficiles à apprécier, en particulier toutes les incertitudes de prélèvements, de neutralisation quand cette dernière est nécessaire, etc. Pour réaliser la comparaison, nous avons donc utilisé l'approche statistique basée sur les tests d'hypothèses. Les quantités que nous souhaitons comparer n'étant pas à priori indépendantes, dans le cas d'un nettoyage correct, nous avons fait le test d'hypothèse suivant : les quantités totales en matières protéique et minérale enlevées par les deux solutions de nettoyage sont égales à celles qu'il y avait à nettoyer. La procédure de réalisation du test est la suivante : nous faisons la différence $D_{i}$ entre le poids total de protéines (ou de minéraux) enlevé par le nettoyage et celui qui était présent avant nettoyage pour tous les essais. L'emploi de ce test suppose que les différences $D_{i}$ entre individus d'une même expérience sont réparties autour d'une moyenne $M_{n}$ qui représente la différence moyenne des deux masses, calculée sur la population dont ces expériences ont été extraites. Il faut également supposer que les écarts $D_{i}-M_{v}$ sont indépendants et normalement distribués autour de $M_{\bar{D}}$ avec une moyenne nulle. En outre, la différence moyenne D observée est normalement distribuée autour de $M_{\mathrm{D}}$ avec un écart type $\sigma_{\mathrm{p}} / \sqrt{n}$ où $\sigma_{\mathrm{n}}$ est l'écart type de la population des différences et $\mathrm{n}$ le nombre 
d'expériences considérées. Une estimation de $\sigma_{\mathrm{p}}$ sur la base de (n-1) degrés de liberté est donnée par :

$$
S \mathrm{~S}=\sqrt{\frac{\left(\mathrm{D}_{1}-\mathrm{D}\right)^{2}}{\mathrm{n}-1}}
$$

Dans le cas des petits échantillons, la distribution de Student (loi de probabilité de Student-Fisher) avec (n-1) degrés de liberté est utilisée pour tester l'hypothèse nulle $\left(M_{v}=0\right)$ : la grandeur $\mathrm{t}=\mathrm{D} \sqrt{\mathrm{n}}\left(\mathrm{S}_{\mathrm{D}}\right)^{-1}$ est comparée à la valeur de la table de "Student Fisher » pour le nombre de degrés de liberté correspondants et pour différents risques d'erreur de "première espèce ". Ce risque correspond à la probabilité de réfuter une hypothèse qui se révèlerait pourtant vraie.

Dans le cas qui nous intéresse, une conclusion positive vise non pas à réfuter l'hypothèse, mais au contraire à l'accepter. Le risque d'erreur encouru, qui est alors un risque de " deuxième espèce " (probabilité d'accepter une hypothèse fausse) est malheureusement impossible à évaluer. On procèdera donc en déterminant le risque de première espèce qui conduit à réfuter l'hypothèse, en visant évidemment un risque important pour pouvoir raisonnablement conclure à l'égalité des masses "à nettoyer » et « effectivement nettoyées ».

\section{Section de préchauffage $E$}

Les résultats sont présentés dans le tableau 1. La méthode statistique décrite précédemment appliquée à l'ensemble des essais de type S-A et A-S (5 essais au total) permet de calculer une valeur de $t$ de 0,8 pour les protéines et de 1,3 pour les minéraux. Ces valeurs conduisent à réfuter l'hypothèse pour des probabilités respectives de 0,46 et 0,27 . Les bilans protéiques et minéraux sont donc satisfaisants ce qui permet de tirer les conclusions suivantes :

- lorsque le nettoyage alcalin est effectué en premier (essais 1, 2 et 3 notés S-A), la totalité des protéines et plus de la moitié des minéraux (58 à $76 \%$ ) sont éliminées par la solution d'hydroxyde de sodium ; le nettoyage acide consécutif enlève la fraction minérale restante ;

- lorsque le nettoyage acide (essais 4 et 5 notés A-S) est effectué en premier, la totalité des minéraux est éliminée sans effet notable sur les protéines qui sont enlevées en totalité par le nettoyage alcalin qui suit. Dans ce cas là, les matières organiques sont donc enlevées préférentiellement par l'hydroxyde de sodium alors que les matières minérales le sont par l'acide nitrique.

Après démontage de l'échangeur, nous constatons visuellement que, dans chaque cas, la totalité des dépôts a été éliminée par l'association du nettoyage alcalin et du nettoyage acide. 


\section{Section de chauffage $F$}

Les résultats sont présentés dans le tableau 2. L'étude statistique appliquée à l'ensemble des essais de type S-A et A-S (5 e ssais au total) permet de calculer une valeur de « $\mathrm{t} »$ de 3,455 pour les protéines et de 0,188 pour les minéraux. Ces valeurs conduisent à réfuter l'hypothèse émise pour des probabilités respectives de 0,025 et 0,87 . Les bilans minéraux sont satisfaisants ce qui permet de tirer les conclusions suivantes :

Quel que soit l'ordre de passage de la solution acide, celle-ci enlève la totalité des minéraux.

Les bilans protéiques par contre ne sont pas satisfaisants. Nous ne retrouvons pas dans les solutions alcalines les quantités de protéines qu'il y avait à enlever. Lorlsque le nettoyage alcalin est effectué en premier (essais 6 et 7), nous retrouvons environ $85 \%$ des protéines dans les deux solutions, mais après démontage, nous remarquons sur les plaques la présence d'un gel aux points de contact des plaques dont l'analyse montre qu'il est formé de protéines.

Lorsque le nettoyage acide est effectué en premier, seulement $45 \%$ des protéines initiales se retrouvent dans les deux solutions. Mais au démontage, l'appareil est propre. Nous supposons qu'il y a eu hydrolyse acide des protéines, en particulier pendant le stockage des échantillons ; or les acides aminés ne sont pas dosables par les méthodes analytiques utilisées.

\section{Etude de l'évolution du taux de colmatage}

Les résultats sont présentés dans le tableau 3.

\section{Section de préchauffage $E$}

L'évolution de la pression différentielle montre que le premier nettoyage, quand il est alcalin (essais 1 à 3 ) permet de réduire considérablement le taux de colmatage. En effet, le taux de colmatage initial, variable entre $8 \%$ et $21 \%$ diminue jusqu'à $2 \%$ qui est une valeur proche de celle observée en fin de nettoyage final sans toutefois lui être égale $\left(\alpha_{\mathrm{r}}=0 \pm 1 \%\right)$. Le taux de colmatage traduisant principalement l'épaisseur de dépôt adhérant aux parois d'échange, nous pouvons en conclure que ce premier nettoyage alcalin enlève la majorité du dépôt en volume. Toutefois, un nettoyage acide reste indispensable pour redonner aux plaques leur aspect initial $\left(\alpha_{\mathrm{r}}=0 \pm 1 \%\right)$.

Lorsque le nettoyage acide est effectué en premier (essais 4 et 5), le taux de colmatage reste globalement constant. La solution acide enlève la majorité des dépôts minéraux sans que l'épaisseur du 
TABLEAU 3

Evolution du taux de colmatage entre le début du nettoyage et la fin du nettoyage pour chaque solution

Evolution of colmation level between beginning and end of the cleaning for each solution

\begin{tabular}{|c|c|c|c|c|c|}
\hline & & \multicolumn{2}{|c|}{ Premier nettoyage } & \multicolumn{2}{|c|}{ Second nettoyage } \\
\hline & & $\begin{array}{l}\alpha_{i}, \% \\
\pm 1 \%\end{array}$ & $\begin{array}{l}\alpha_{\mathrm{p}}, \% \\
\pm 1 \%\end{array}$ & $\begin{array}{l}\alpha_{i}, \quad \% \\
\pm 1 \%\end{array}$ & $\begin{array}{l}\alpha_{\mathrm{f}}, \% \\
\pm 1 \%\end{array}$ \\
\hline \multirow{5}{*}{ 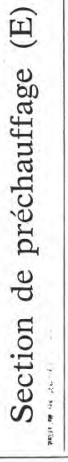 } & $\begin{array}{l}\text { Essai } 1 \\
\text { S-A }\end{array}$ & 21,4 & 2,2 & 3,7 & 0 \\
\hline & $\begin{array}{l}\text { Essai } 2 \\
\text { S-A }\end{array}$ & 13,8 & 2,3 & 3,4 & 0 \\
\hline & $\begin{array}{l}\text { Essai } 3 \\
\text { S-A }\end{array}$ & 8,1 & 2,1 & 2,6 & 0 \\
\hline & $\begin{array}{l}\text { Essai } 4 \\
\text { A-S }\end{array}$ & 15,0 & 18,0 & 17,3 & .0 \\
\hline & $\begin{array}{l}\text { Essai } 5 \\
\text { A-S }\end{array}$ & 17,3 & 19,3 & 17,8 & 0 \\
\hline \multirow{5}{*}{ 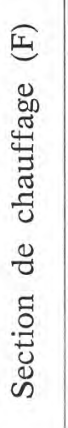 } & $\begin{array}{l}\text { Essai } 6 \\
\text { S-A }\end{array}$ & 16,8 & 15,0 & 14,0 & 1,1 \\
\hline & $\begin{array}{l}\text { Essai } 7 \\
\text { S-A }\end{array}$ & 5,0 & 14,4 & 15,9 & 1,0 \\
\hline & $\begin{array}{l}\text { Essai } 8 \\
\text { A-S }\end{array}$ & 8,3 & 6,7 & 6,2 & 0 \\
\hline & $\begin{array}{l}\text { Essai } 9 \\
\text { A-S }\end{array}$ & 11,2 & 8,2 & 7,6 & 0 \\
\hline & $\begin{array}{l}\text { Essai } 10 \\
\text { A-S }\end{array}$ & 12,8 & 11,4 & 11,5 & 0 \\
\hline
\end{tabular}

$\alpha_{i}$, taux de colmatage avant nettoyage ; $\alpha_{\mathrm{f}}$, taux de colmatage en fin de nettoyage ; S-A, nettoyage alcalin suivi du nettoyage acide ; A-S, nettoyage acide suivi du nettoyage alcalin.

$\alpha_{i}$, colmation level before cleaning ; $\alpha_{f}$,colmation level after cleaning ; $S-A$, alkaline cleaning followed by acid cleaning ; $A-S$, acid cleaning followed by alkaline cleaning. 


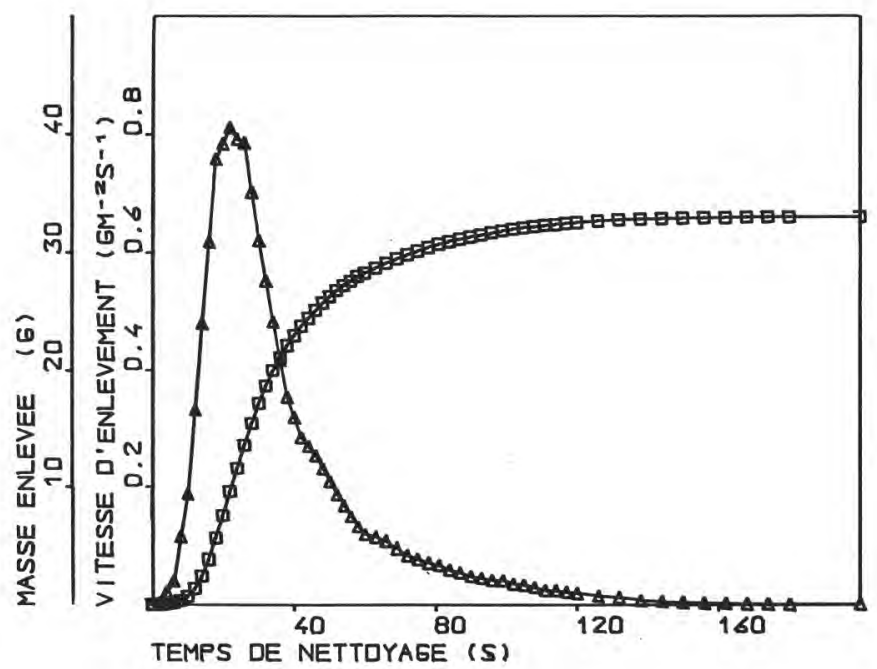

FIG. 4

Evolution de la vitesse d'enlèvement des protéines et de la masse enlevée par la solution alcaline en fonction du temps de nettoyage; $\triangle$, vitesse d'enlèvement des protéines; $\square$, masse de protéines enlevées.

Evolution of rate amount of protein removed by the alkaline solution versus cleaning time; $\triangle$, protein removing rate; $\square$, mass of removed protein.

dépôt varie. Le nettoyage alcalin qui suit enlève les matières organiques et réduit l'épaisseur du dépôt à zéro.

\section{Section de chauffage $F$}

Le nettoyage alcalin effectué en premier (essais 6 et 7) n'entraîne pas une diminution du taux de colmatage mais, dans un cas sur deux, il entraîne un gonflement du dépôt qui ne diminue pas quel que soit le temps de passage de cette solution. Pour un taux de colmatage initial compris entre $5 \% \pm 1 \%$ et $16,8 \% \pm 1 \%$ (essais 6 et 7), le taux de colmatage final atteint $14 \% \pm 1 \%$ et $15 \% \pm 1 \%$. Le nettoyage acide qui suit ne permet pas l'élimination totale des dépôts et un gel translucide reste aux points de contacts des plaques. Le taux de colmatage est égal à $1 \% \pm 1 \%$ pour les 2 essais. Toutefois, une expérience de nettoyage à l'échelle industrielle effectuée dans les mêmes conditions mais à $140^{\circ} \mathrm{C}$ (nettoyage intermédiaire) ne conduit à la formation d'aucun gel protéique. 


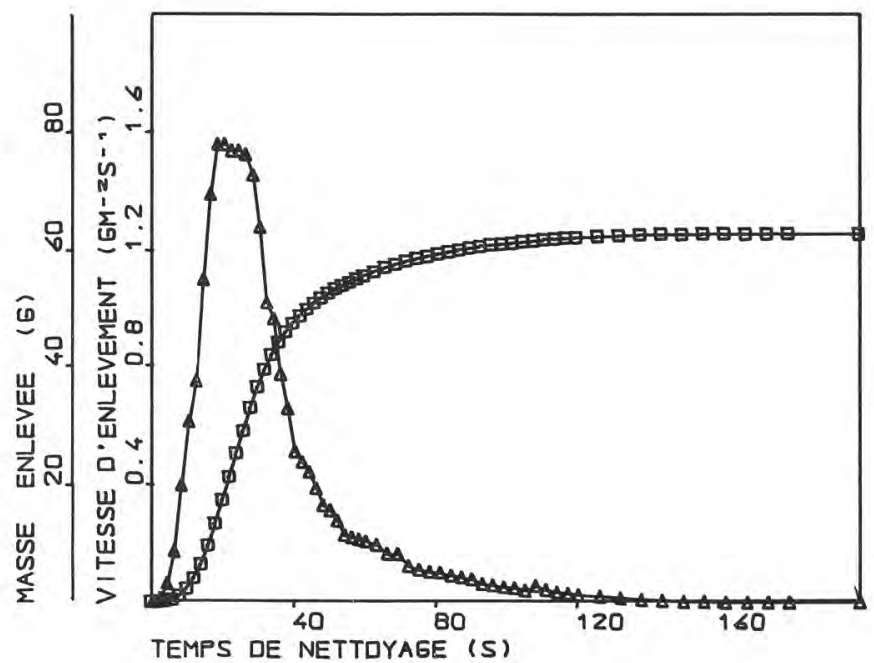

FIG. 5

Evolution de la vitesse d'enlèvement des minéraux et de la masse enlevée par la solution acide en fonction du temps de nettoyage; $\triangle$, vitesse d'enlèvement des minéraux; $\square$, masse minérale enlevée.

Evolution of rate and amount of minerals removed by the acid solution versus cleaning time; $\triangle$, minerals removing rate; $\square$, mass of removed minerals.

Il semble donc que l'apparition d'un tel gel dépende des conditions opératoires de nettoyage.

Par contre, si le nettoyage acide est effectué avant le nettoyage alcalin, le taux de colmatage initial compris entre $8 \% \pm 1 \%$ et $13 \% \pm 1 \%$ diminue légèrement (environ 1 à $2 \%$ ). Et à l'issue du nettoyage, les plaques d'échange sont propres.

Les petites différences observées entre le taux de colmatage final du premier nettoyage et le taux de colmatage initial du second nettoyage sont essentiellement dues aux imprécisions de mesure du taux de colmatage lors du rinçage.

\section{Etude de la vitesse d'enlèvement des dépôts}

L'évolution de la vitesse d'enlèvement des dépôts, en terme d'apparition de la souillure dans l'effluent de nettoyage en fonction de la durée de nettoyage est décrite par les figures 4 et 5 . 


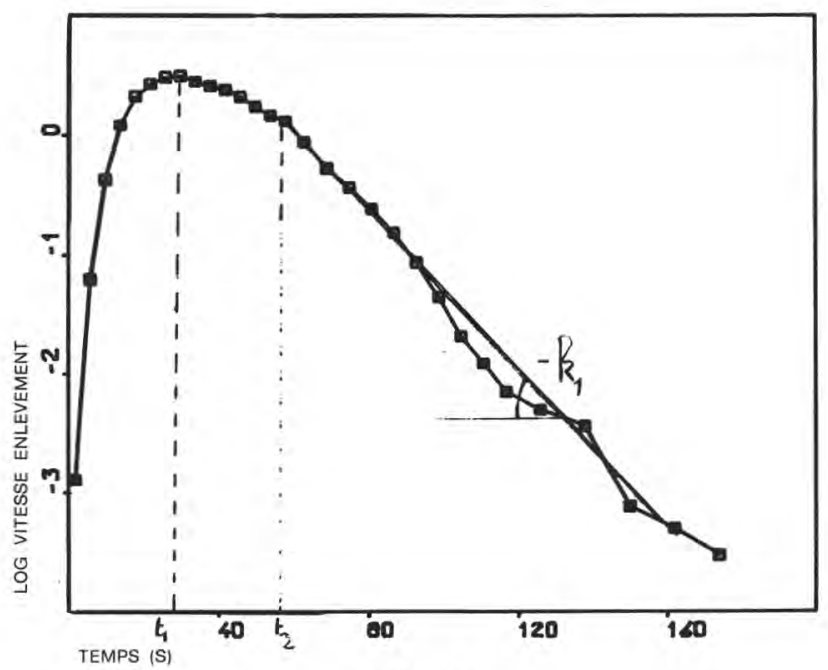

FIG. 6

Détermination graphique des paramètres cinétiques $k_{1}, t_{i}, t_{2}$ à partir de la courbe d'évolution du logarithme népérien de la vitesse d'enlèvement en fonction du temps ; $k_{1}$ constante de vitesse d'ordre 1 en $s^{-1} ; t_{1}$ temps $(s)$ pour lequel le logarithme de la vitesse d'enlèvement est maximum; $t_{2}$, temps à partir duquel le logarithme de la vitesse d'enlèvement devient linéaire.

Graphical determination of kinetic parameters $k_{1}, t_{1}, t_{2}$ from logarithmical evolution curve of the removing rate versus cleaning time; $k_{1}$ in $s^{-1}$, velocity constant of 1 st order; $t_{1}$, time in $s$ where the removing rate is maximum; $t_{2}$, time in $s$ where the logarithmical evolution curve becomes linear.

La figure 4 représente d'une part la vitesse d'enlèvement des protéines et d'autre part la masse de protéines enlevées par l'effluent alcalin en fonction de temps de nettoyage (calculée comme étant l'intégrale de la précédente). La vitesse de nettoyage apparente est nulle au temps initial, puis elle croît jusqu'à un maximum et enfin décroît plus ou moins rapidement jusqu'à zéro. Cette décroissance, après une partie linéaire de plus ou moins courte durée, est de forme exponentielle comme le montre le tracé en coordonnée logarithmique de la vitesse d'enlèvement en fonction du temps (fig. 6). La masse enlevée (en gramme de matières sèches) est représentée par une sigmoïde, qui part de zéro au temps initial et se termine par un plateau horizontal lorsque toute la masse a été enlevée. De la même manière, la figure 5 représente la vitesse d'enlèvement des minéraux et la masse minérale enlevée en milieu acide en fonction du temps de nettoyage. Les allures observées sont exactement les mêmes que pour les protéines en milieu alcalin.

Ces courbes montrent donc que quel que soit l'élément considéré et quelle que soit la solution détergente utilisée la vitesse de 


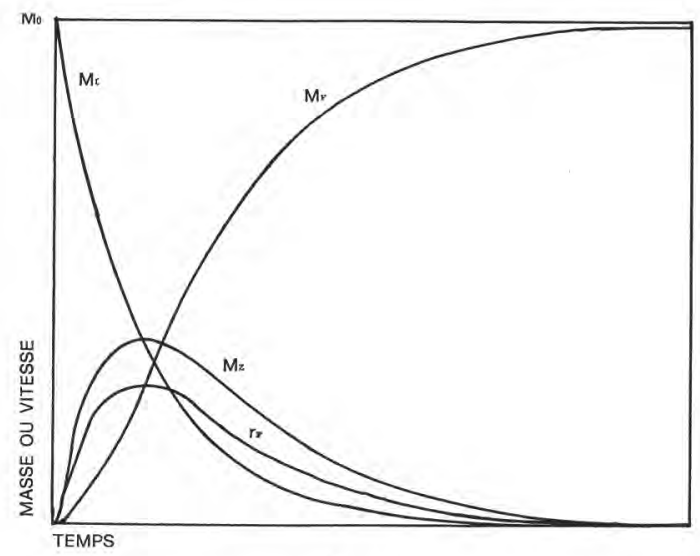

FIG. 7

Evolution des masses des différents composés et de la vitesse d'apparition du composé final correspondant à un schéma réactionnel composé d'une réaction d'ordre 0 vis-à-vis de la masse du composé initial en série avec une réaction d'ordre 1 vis-à-vis de la masse du composé intermédiaire. Mo, masse initiale à nettoyer en $g ; M_{l}$, masse du composé initial en $g ; M_{Z}$, masse du composé intermédiaire en $g ; M_{F}$, masse du composé finah en $g ; r_{F}$, vitesse d'apparition $d u$ composé final en g. $m^{-2} s^{-1}$.

Mass evolution of different compounds and apparition rate curve of final compound. Mo, initial total dry matter in $g ; M_{I}$, mass of initial compound in $g$; $M_{z}$, mass of intermediary compound in $g ; M_{F}$, mass of final compound in $g$;

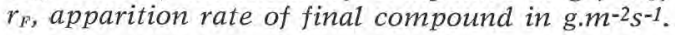

nettoyage apparente présente la même évolution. L'allure de nos courbes est analogue aux résultats de GALLOT-LAVALLÉE (1982) obtenus sur des portions de tubulures encrassées, bien que nos essais portent sur une surface d'échange environ 100 fois plus importante aussi bien pour la section de chauffage $F$ que pour la section de préchauffage $\mathrm{E}$.

L'allure des courbes expérimentales observée élimine tout mécanisme ne comportant qu'une seule étape réactionnelle. C'est pourquoi, Gallot-LAVAlléE (1982) propose un schéma réactionnel comportant deux étapes limitantes en série la première d'ordre 0 et la deuxième d'ordre 1 ; les ordres de réaction sont définis par rapport à la masse de dépôt. Ce schéma peut se définir de la manière suivante :

$\begin{array}{ccccc}\text { I } \\ \begin{array}{c}\text { initial } \\ k_{0}\end{array} & \text { Remposé } & \begin{array}{c}\text { composé } \\ \text { intermédiaire }\end{array} & k_{1} & \begin{array}{c}\text { dépôt en solution } \\ \text { ou suspension } \\ \text { dans la solution } \\ \text { de nettoyage }\end{array}\end{array}$


Dans ce schéma, la masse de ce composé initial décroît linéairement en fonction du temps. L'étape réactionnelle limitante étant toujours la seconde étape, la vitesse d'apparition du composé intermédiaire $\mathrm{Z}$ est dans un premier temps supérieure à sa vitesse de disparition. La masse de ce composé intermédiaire est donc nulle au temps 0 , puis augmente jusqu'à un maximum qui correspond au temps $t_{1}$ pour lequel il n'y a plus de dépôt initial dans l'appareil. A partir de ce temps, il n'y a plus que la seconde étape réactionnelle à considérer et la masse du dépôt intermédiaire décroît donc exponentiellement en fonction du temps. La masse du composé final vaut zéro au temps zéro puis augmente selon une allure sigmoïde jusqu'à une valeur finale égale à celle de la masse initiale de dépôt à nettoyer. La vitesse d'apparition du composé final a une allure identique à la masse du composé intermédiaire, ces deux grandeurs étant proportionnelles (fig. 7). Par conséquent, la vitesse d'apparition de la souillure dans la solution détergente croît jusqu'au temps $t_{1}$ pour lequel elle présente une discontinuité puis elle décroît exponentiellement et tend vers zéro.

L'application de ce schéma réactionnel à notre cas pose le problème de la répartition de la masse de dépôt initial sur la surface d'échange. En effet, elle n'est pas uniformément répartie, comme c'est le cas pour les tubulures étudiées par Gallot-LavalléE (1982)

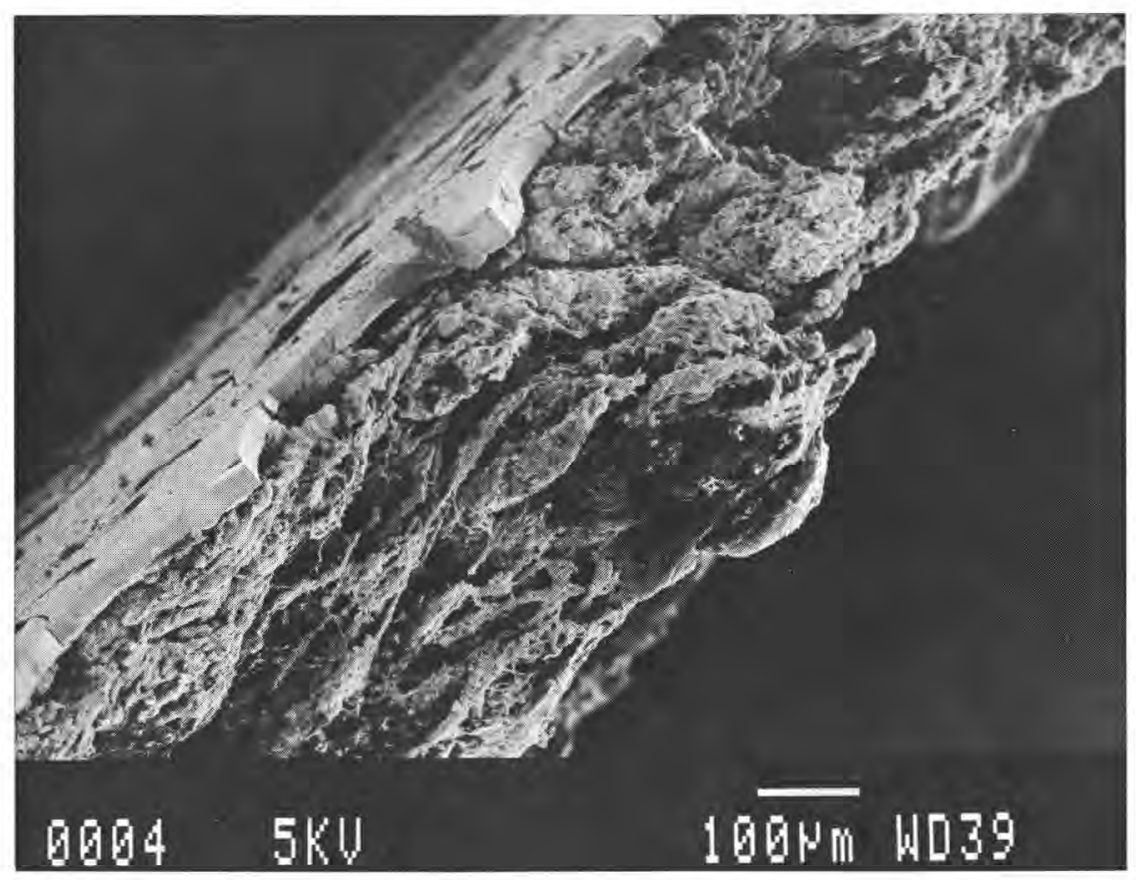

Vue en coupe du dépôt de la section du préchauffage E effectuée au microscope électronique à balayage. 
mais augmente de l'entrée à la sortie de la section considérée. Cette particularité implique que l'évolution de la vitesse de nettoyage citée précédemment est en partie modifiée. En particulier, le maximum de vitesse observé pour le temps $t_{1}$ ne correspond plus à l'épuisement du composé initial mais au moment où une partie de la surface d'échange ne comporte déjà plus de ce composé initial alors qu'il est encore présent dans les endroits initialement les plus encrassés. L'allure observée présente donc une partie intermédiaire correspondant à une phase où nous ne sommes plus totalement en présence des deux réactions en série et pas encore en présence d'une seule réaction, ceci en considérant l'ensemble de la surface. Le temps $\mathrm{t}_{2}$ à partir duquel il n'y a plus de dépôt initial sur l'ensemble de la surface est donc en fait supérieur au temps $t_{1}$ correspondant au maximum de la vitesse. Pour régler ce problème, nous proposons de considérer en première approximation que la vitesse d'enlèvement que l'on observerait si le dépôt était régulièrement réparti sur la surface suit une loi exponentielle à partir du temps $t_{x}$ que nous définissons comme la moyenne arithmétique entre les temps $t_{1}$ et $t_{2}$.

A partir de ce raisonnement, il est possible de définir, en tenant compte de la seule expérience, les deux constantes de vitesse $\mathbf{k}_{\mathrm{o}}$ et $\mathbf{k}_{1}$. La procédure utilisée est la suivante : nous déterminons graphiquement en prenant la pente de la partie linéaire du tracé du logarithme de la vitesse en fonction du temps (fig. 6), le point de cassure de cette droite qui correspond au temps $t_{2}$. En prenant le maximum $\mathrm{du}$ logarithme de la vitesse, nous déterminons le temps $t_{1}$. Nous estimons alors la constante de vitesse d'ordre $\mathrm{O}$ par la relation suivante :

$$
\mathrm{k}_{\mathrm{o}}=\frac{\mathrm{M}_{\mathrm{o}}}{\mathrm{t}_{\mathrm{x}} \mathrm{S}}
$$

où $\mathrm{k}_{\mathrm{o}}$ est exprimé en g.m $\mathrm{m}^{-2} \mathrm{~s}^{-1}, \mathrm{t}_{\mathrm{x}}$ est défini par $\left(\mathrm{t}_{1}+\mathrm{t}_{2}\right) / 2$ en secondes, $\mathbf{M}_{0}$ est la masse totale nettoyée en $\mathrm{g}$ et $\mathrm{S}$ la surface totale nettoyée en $\mathrm{m}^{2}$.

A partir de $t_{2}$, la masse $M_{z}$ du composé intermédiaire décroît exponentiellement selon la relation :

$$
\begin{aligned}
& M_{z}=C \exp \left(-k_{1} t\right) \\
& \text { avec } t>t_{2}
\end{aligned}
$$

et $\mathrm{C}$ masse de dépôt restant à nettoyer au temps $\mathrm{t}_{2}$.

La vitesse de nettoyage du dépôt, définie comme étant l'opposé de la dérivée de la masse de composé intermédiaire par rapport au temps $\left(\mathrm{v}=-\mathrm{dM}_{\mathrm{z}} / \mathrm{dt}\right.$ ) est alors telle que :

$$
\text { Ln } \mathrm{v}=\text { constante }-\mathrm{k}_{1} \mathrm{t}
$$

La constante de vitesse $\mathrm{k}_{1}$ est donc déterminée par la pente expérimentale de cette droite.

Nous pouvons ainsi dans tous les cas, où les bilans matières sont corrects, déterminer en première approximation les constantes de vitesse d'ordre 0 et d'ordre 1 du modèle de Gallot-LavalléE (1982). 
Ceci nous a permis de comparer les constantes de vitesse obtenues pour le nettoyage des minéraux et des protéines lorsque les procédures de nettoyage de type S-A et de type A-S ont été appliquées soit à la zone $\mathrm{E}$ soit à la zone $\mathrm{F}$. Pour réaliser cette comparaison, nous avons également utilisé l'approche statistique basée sur les tests d'hypothèse. Les quantités que nous voulons comparer étant a priori indépendantes dans le cas de nettoyages corrects, nous avons fait le test d'hypothèse suivant : les constantes de vitesse obtenues pour les nettoyages de type S-A sont égales à celles obtenues pour les nettoyages de type A-S.

La procédure de réalisation du test est la suivante : lorsque les quantités de matières nettoyées et celles qui étaient à nettoyer sont égales, pour une zone et une solution déterminées, nous faisons la moyenne des constantes de vitesse d'ordre 0 (ou d'ordre 1) des nettoyages où nous avons utilisé la procédure S-A ainsi que celle des constantes de vitesse d'ordre 0 (ou d'ordre 1 ) où nous avons employé la procédure A-S.

L'emploi de ce test suppose que les deux échantillons d'effectif $\mathrm{n}_{1}$ et $\mathrm{n}_{2}$ ayant comme moyenne respective $\mathrm{X}_{1}$ et $\mathrm{X}_{2}$ sont indépendants et que ces moyennes donnent une estimation des moyennes $\mathbf{M}_{1}$ et $\mathbf{M}_{2}$ des deux populations dont les échantillons sont extraits. Il faut également supposer que les écarts $\mathrm{X}_{1}-\mathrm{M}_{1}$ et $\mathrm{X}_{2}-\mathrm{M}_{2}$ sont indépendants et normalement distribués autour de $\mathbf{M}_{1}$ et $\mathbf{M}_{2}$ avec une moyenne nulle. En outre $\mathrm{X}_{1}$ (ou $\mathrm{X}_{2}$ ) est normalement distribuée autour de $\mathrm{M}_{1}$ (ou $\mathrm{M}_{2}$ ) avec un écart type $\sigma \mathrm{x}_{1}-\mathrm{x}_{2}$.

Une estimation de $\sigma \mathrm{x}_{1}-\mathrm{x}_{2}$ est donnée par :

$$
\left.\mathrm{Sx}_{1}-\mathrm{x}_{2}=\frac{\mathrm{s}^{2}}{\left(-\frac{\mathrm{n}_{1}}{\mathrm{n}_{1}}\right.}+\frac{\mathrm{s}_{2}}{-}\right)^{1 / 2}
$$

où $n_{1}$ et $n_{2}$ sont les effectifs de chacun des échantillons considérés et s l'écart type pondéré de ces deux échantillons. Une estimation de $\mathrm{s}^{2}$ est donnée par :

$$
\begin{aligned}
s^{2} & =\frac{\left(\varepsilon X_{1}^{2}+\varepsilon X_{2}^{2}\right)}{n_{1}+n_{2}} \\
\text { où } X_{i}^{2} & =\left(A_{i j}-X_{i}\right)^{2}-\frac{\left(A_{i j}-X_{i}\right)^{2}}{n_{i}}
\end{aligned}
$$

où $A_{i j}$ est le jieme élément de l'échantillon $i$.

La distribution de Student (loi de probabilité de Student-Fisher) pour (n-1) degrés de liberté où $n$ est égal à la plus petite valeur de $\mathrm{n}_{1}$ et de $\mathrm{n}_{2}$ est utilisée pour tester l'hypothèse de nullité $\left(M_{1}=M_{2}=0\right)$.

La grandeur $\mathrm{t}=\left(\mathrm{X}_{1}-\mathrm{X}_{2}\right) / \mathrm{Sx}_{1}-\mathrm{X}_{2}$ est comparée à celle de la table de Student-Fisher pour le nombre de degrés de liberté correspondant 
et pour différents risques d'erreurs de " première espèce ». Dans notre cas, nous cherchons à réfuter l'hypothèse : les constantes de vitesse ne sont pas significativement différentes selon l'ordre de passage des solutions de nettoyage et selon la zone considérée.

\section{Section de préchauffage $E$}

Les résultats sont présentés dans le tableau 4 et classés par type de nettoyage réalisé :

\section{a) Nettoyages de type $S$-A (essais 1, 2 et 3)}

Rappelons que pour ce type de nettoyage, la solution alcaline enlève toutes les matières protéiques mais aussi une fraction des matières minérales et que la solution acide élimine les minéraux restants. Dans le cas de l'enlèvement des dépôts protéiques en milieu alcalin, la constante moyenne de vitesse de la réaction d'ordre 1 est $\mathrm{k}_{1}=0,066 \mathrm{~s}^{-1}$ et celle de la réaction d'ordre 0 est $\mathrm{k}_{0}=0,22 \mathrm{~g} \cdot \mathrm{m}^{-2} \mathrm{~s}^{-1}$. Le temps $\mathrm{t}_{\mathrm{x}}$ à partir duquel il $\mathrm{n}^{\prime} \mathrm{y}$ a plus de protéines à l'état initial correspond à l'enlèvement de $95 \% \pm 5 \%$ de la masse protéique qu'il y avait initialement à nettoyer. Pour l'enlèvement des minéraux en milieu acide, la constante moyenne de vitesse de la réaction d'ordre 0 est $k_{0}=0,28 \mathrm{~g} \cdot \mathrm{m}^{-2} \mathrm{~s}^{-1}$ et celle de la réaction d'ordre 1 est $\mathrm{k}_{1}=0,096 \mathrm{~s}^{-1}$. Le temps $\mathrm{t}_{\mathrm{x}}$ pour lequel tous les minéraux ne sont plus dans leur état initial correspond à l'enlèvement de $60 \% \pm 3 \%$ de la masse minérale nettoyée. Pour l'enlèvement des minéraux en milieu alcalin, les constantes moyennes de vitesse d'ordre 0 et 1 sont respectivement de $0,134 \mathrm{~g} \cdot \mathrm{m}^{-2} \mathrm{~s}^{-1}$ et de $0,0085 \mathrm{~s}^{-1}$. Le temps $t_{x}$ correspond à l'enlèvement de $60 \% \pm 5 \%$ de l'ensemble des minéraux enlevés.

\section{b) Nettoyages de type AS (essais 4 et 5)}

Dans le cas de ce type de nettoyage, la solution acide élimine exclusivement les matières minérales et la solution alcaline exclusivement les matières protéiques. Pour l'enlèvement des protéines en milieu alcalin, les constantes moyennes de vitesse de réaction d'ordre 1 et 0 sont respectivement $k_{1}=0,429 \mathrm{~s}^{-1}$ et $\mathrm{k}_{\mathrm{o}}=1,20 \mathrm{~g}$ $\mathrm{m}^{-2} \mathrm{~s}^{-1}$ et le temps $t_{\mathrm{x}}$ à partir duquel il n'y a plus de protéines dans leur état initial correspond à l'enlèvement de $95 \% \pm 5 \%$ de la masse totale nettoyée. Dans le cas des minéraux, les constantes moyennes de vitesse des réactions d'ordre 0 et 1 sont respectivement $\mathrm{k}_{\mathrm{o}}=0,44 \mathrm{~g} \cdot \mathrm{m}^{-2} \mathrm{~s}^{-1}$ et $\mathrm{k}_{1}=0,03 \mathrm{~s}^{-1}$. Le temps $\mathrm{t}_{\mathrm{x}}$ à partir duquel il n'y a plus de dépôt à l'état initial correspond à l'enlèvement de $60 \% \pm 3 \%$ de la masse totale à enlever.

Pour pouvoir comparer l'efficacité relative des deux types de nettoyage, nous avons appliqué la procédure statistique déjà utilisée aux résultats précédents, en nous limitant à une comparaison des vitesses d'enlèvement des protéines en milieu alcalin et des vitesses d'enlèvement des minéraux en milieu acide. Dans le cas de l'enlè- 
TABLeau 4

Aspect cinétique de l'enlèvement des protéines et des minéraux en milieu alcalin et en milieu acide pour les dépôts de section de préchauffage $E$

Kinetic approach of protein and minerals removal by alkaline and acid solutions in the case of deposits of preheating section $(E)$

\begin{tabular}{|c|c|c|c|c|c|c|c|c|c|c|c|c|}
\hline & \multicolumn{4}{|c|}{ Protéines en milieu alcalin } & \multicolumn{4}{|c|}{ Minéraux en milieu acide } & \multicolumn{4}{|c|}{ Minéraux en milieu alcalin } \\
\hline & $\mathrm{X}, \%$ & $t_{x}, s$ & $\begin{array}{c}K_{0}, \\
\text { g.m } m^{-2} S^{-1}\end{array}$ & $\mathrm{~K}_{1}, \mathrm{~s}^{-1}$ & $\mathrm{X}, \%$ & $t_{x}, s$ & $\underset{\text { g.m }{ }^{-2} \mathrm{~s}^{-1}}{\mathrm{~K}_{\mathrm{i}}}$ & $\mathrm{K}_{1}, \mathrm{~s}^{-1}$ & $\mathrm{X}, \%$ & $t_{x}, s$ & $\underset{\text { g.m }}{\mathrm{K}_{\mathrm{o}},}$ & $\mathrm{K}_{1}, \mathrm{~s}^{-1}$ \\
\hline $\begin{array}{l}\text { Essai } 1 \\
\text { S-A }\end{array}$ & 90 & 210 & 0,222 & 0,0726 & 58 & 19 & 0,280 & 0,0935 & 60 & 120 & 0,217 & 0,0095 \\
\hline $\begin{array}{l}\text { Essai } 2 \\
\text { S-A }\end{array}$ & 95 & 164 & 0,226 & 0,0660 & 60 & 24 & 0,278 & 0,0846 & 60 & 129 & 0,094 & 0,0132 \\
\hline $\begin{array}{l}\text { Essai } 3 \\
\text { S-A }\end{array}$ & 95 & 120 & 0,210 & 0,0594 & 60 & 21 & 0,271 & 0,1100 & 59 & 60 & 0,091 & 0,0368 \\
\hline $\begin{array}{l}\text { Essai } 4 \\
\text { A-S }\end{array}$ & 95 & 83 & 0,695 & 0,425 & 61 & 56 & 0,410 & 0,0209 & 61 & 41 & 0,0092 & 0,0564 \\
\hline $\begin{array}{l}\text { Essai } 5 \\
\text { A-S }\end{array}$ & 94 & 52 & 0,510 & 0,430 & 60 & 33 & 0,466 & 0,0357 & 56 & 34. & 0,0077 & 0,0713 \\
\hline
\end{tabular}

S-A, nettoyage alcalin suivi du nettoyage acide ; A-S, nettoyage acide suivi du nettoyage alcalin ; S, surface nettoyée égale à $1,275 \mathrm{~m}^{2} ; \mathrm{X}$, pourcentage de la masse totale enlevée par la solution considérée au temps $t_{x} ; t_{x}$, temps à partir duquel la vitesse de nettoyage deviendrait exponentielle si la répartition était uniforme.

$S-A$, alkaline cleaning followed by acid cleaning; $A-S$, acid cleaning followed by alkaline cleaning ; $S$, cleaned surface (equal to $1.275 \mathrm{~m}^{2}$ ); $X$, percentage of total removed mass by the considered solution at time $t_{x} ; t_{x}$, time where the cleaning rate would become exponential if the deposits repartition was uniform. 
vement des protéines en milieu alcalin, nous calculons des valeurs de $« t$ » de 5,53 pour la constante de vitesse d'ordre 0 et de 63,1 pour celle d'ordre 1 . Avec des risques d'erreur respectivement de $5 \%$ et de $1 \%$, nous en déduisons que les constantes de vitesse d'ordre 0 et d'ordre 1 sont significativement différentes selon l'ordre de passage de la solution alcaline. Elles sont respectivement 5,5 et 6,5 fois plus importantes pour les essais de type A-S c'est-à-dire lorsque le nettoyage acide est effectué en premier.

Dans le cas de l'enlèvement des minéraux par la solution acide, les valeurs de « $t$ » obtenues sont de 7,65 pour la constante de vitesse d'ordre 0 et de 6,1 pour celle d'ordre 1 . Ces valeurs conduisent, avec des risques d'erreur de $2 \%$ dans les deux cas, à considérer que des constantes de vitesse d'ordre 0 et 1 sont là encore significativement différentes. Toutefois comparé au cas précédent seule la constante de vitesse d'ordre 0 est augmentée (multipliée par 1,6) dans le cas des nettoyages de type A-S. Par contre, la constante de vitesse d'ordre 1 est 0,3 fois plus faible que pour les nettoyages de type S-A.

En résumé, nous pouvons donc dire que pour la section de préchauffage E dont le dépôt est plutôt organique, les vitesses d'enlèvement des protéines en milieu alcalin sont nettement accélérées Iorsque la solution acide est utilisée en premier. Pour l'enlèvement des minéraux en milieu acide, chacune des procédures de nettoyage privilégie une phase différente de la réaction de nettoyage. La deuxième étape, celle d'ordre 1, étant l'étape limitante, le fait qu'elle soit ralentie dans le cas des nettoyages de type A-S ne permet pas de conclure sur l'avantage global de cette procédure. C'est pourquoi nous avons ultérieurement introduit les notions de temps utile et de durée totale efficace de nettoyage.

\section{Section de chauffage $F$}

Rappelons d'une part que les dépôts formés dans cette section sont essentiellement minéraux $(70 \%)$ et d'autre part que les bilans (entre les quantités de dépôt adhérant aux plaques et les quantités de dépôt récupéré dans les solutions de nettoyage) réalisés sur les différents constituants ne conduisent pas à des résultats aussi satisfaisants que pour le tronçon de préchauffage de l'échangeur. En particulier, et quelles qu'en soient les causes (procédure de nettoyage incomplète, ou dégradation des constituants lors du stockage des solutions de nettoyage) le bilan en matières protéiques n'est pas satisfaisant. En conséquence, nous nous sommes limités à une comparaison des critères de nettoyage seulement pour l'élimination des minéraux en milieu acide.

Les résultats sont présentés dans de tableau 5 .

Dans le cas des nettoyages de type S-A (essais 6 et 7), les valeurs moyennes des constantes de vitesse des réactions d'ordre 0 


\section{TABLEAU 5}

Aspect cinétique de l'enlèvernent des minéraux par la solution acide pour la section de chauffage $F$

Kinetic approach of minerals removal by acid solution in the case of deposits of heating section (F)

\begin{tabular}{l|c|c|c|c}
\hline & \multicolumn{3}{|c}{ Minéraux en milieu acide } \\
\cline { 2 - 4 } & $\mathrm{X}, \%$ & $\mathrm{t}_{\mathrm{x}}, \mathrm{s}$ & $\mathrm{K}_{0}, \mathrm{mg}^{-2} \mathrm{~s}^{-1}$ & $\mathrm{~K}_{1}, \mathrm{~s}^{-1}$ \\
\hline $\begin{array}{l}\text { Essai 6 } \\
\text { S-A }\end{array}$ & 51 & 53 & 1,459 & 0,0205 \\
$\begin{array}{l}\text { Essai 7 } \\
\text { S-A }\end{array}$ & 40 & 37 & 1,172 & 0,0198 \\
$\begin{array}{l}\text { Essai } \\
\text { A-S }\end{array}$ & 49 & 33 & 0,886 & 0,016 \\
$\begin{array}{l}\text { Essai } \\
\text { A-S }\end{array}$ & 40 & 43 & 1,020 & 0,017 \\
$\begin{array}{l}\text { Essai 10 } \\
\text { A-S }\end{array}$ & 40 & 45 & 0,945 & 0,018 \\
\hline
\end{tabular}

S-A, nettoyage alcalin suivi du nettoyage acide; A-S, nettoyage acide suivi du nettoyage alcalin ; $\mathrm{S}$, surface nettoyée égale à $1,200 \mathrm{~m}^{2}$; X, pourcentage de la masse totale enlevée par la solution acide au temps $t_{x} ; t_{x}$, temps à partir duquel la vitesse de nettoyage deviendrait exponentielle si la répartition était uniforme.

S-A, alkaline solution followed by acid solution ; A-S, acid solution followed by alkaline solution; $S$, cleaned surface (equal to $1.200 \mathrm{~m}^{2}$ ) ; $X$, percentage of total removed mass by the acid solution at time $t_{x} ; t_{x}$, time where the cleaning rate would become exponential if the deposits repartition was uniform.

et 1 sont respectivement $k_{0}=1,3 \mathrm{~g} \cdot \mathrm{m}^{-2} \mathrm{~s}^{-1}$ et $\mathrm{k}_{1}=0,0201 \mathrm{~s}^{-1}$. Le temps $t_{x}$ pour lequel il n'y a plus de minéraux à l'état initial correspond au nettoyage de $40 \% \pm 10 \%$ du dépôt minéral.

Pour la procédure de type A-S, c'est-à-dire lorsque le nettoyage acide est effectué en premier (essais 8, 9 et 10), les valeurs moyennes des constantes de vitesse des réactions d'ordre 0 et 1 sont respectivement de $k_{0}=0,95$ g.m $\mathrm{m}^{-2} \mathrm{~s}^{-1}$ et $\mathrm{k}_{1}=0,017 \mathrm{~s}^{-1}$. Le temps $\mathbf{t}_{\mathrm{x}}$ observé dans ce cas est le même que pour la procédure S-A.

L'étude statistique précédemment décrite permet de calculer les valeurs de « $\mathrm{t}$ » de 3,10 pour la constante de vitesse d'ordre 0 et de 3,62 pour celle d'ordre 1 . Avec un risque d'erreur de première espèce 
de $5 \%$, ces résultats conduisent à la conclusion que les valeurs des constantes de vitesse sont significativement différentes. Le passage de la solution alcaline accélère légèrement les vitesses d'enlèvement des minéraux par l'acide (les constantes de vitesse d'ordre 0 et d'ordre 1 sont respectivement 1,4 fois et 1,2 fois plus fortes) mais pose par ailleurs d'autres problèmes importants tels que la formation d'un gel protéique restant aux points de contact des plaques d'échange à la fin du nettoyage complet.

\section{E. Etude du temps utile et des durées, efficace et spécifique de nettoyage}

Nous définissons pour chaque nettoyage réalisé le temps utile de nettoyage comme le temps nécessaire pour que la vitesse de nettoyage ne soit plus mesurable par les méthodes analytiques. Nous définissons également, pour chaque procédure complète utilisée, la durée efficace comme la somme des durées utiles de chaque nettoyage élémentaire et la durée spécifique comme la somme des durées utiles ramenées à la masse de matières enlevées par la solution considérée.

\section{Section de préchauffage $E$}

Les résultats sont présentés dans le tableau 6. Le temps utile de nettoyage par la seconde solution de nettoyage est toujours inférieur à celui de la première solution sauf pour l'essai 9 où nous observons l'inverse. Mais la durée spécifique de nettoyage varie de la façon suivante : lorsque la solution alcaline est utilisée en premier (essais 1, 2 et 3), elle est comprise entre $10,0 \mathrm{~s} . \mathrm{g}^{-1}$ et $21,7 \mathrm{~s} . \mathrm{g}^{-1}$ et lorsque la solution alcaline est utilisée en second (essais 4 et 5 ), elle est comprise entre $7,6{\mathrm{~s} . \mathrm{g}^{-1}}^{\text {et } 9,1} \mathrm{s.g} \mathrm{g}^{-1}$ alors que les masses à nettoyer varient dans la même plage (50 g à $130 \mathrm{~g})$.

L'étude statistique basée sur les tests d'hypothèse montre que la différence entre les moyennes de deux échantillons indépendants est significative avec un risque d'erreur de $15 \%$. Or dans le cas où le nettoyage acide est effectué en premier, la durée moyenne spécifique de nettoyage est de $8,4 \mathrm{s.g}^{-1}$, alors que dans l'autre cas, elle est de $17 \mathrm{s.g}{ }^{-1}$. Nous constatons donc que les vitesses de nettoyage spécifiques sont multipliées par deux lorsque le nettoyage alcalin est effectué en second. Ce résultat va dans le même sens que celui que nous obtenons par l'étude des vitesses d'enlèvement.

\section{Section de chauffage $F$}

Les résultats sont présentés dans le tableau 6. La durée de nettoyage spécifique est très variable d'un essai à l'autre et elle ne peut pas nous permettre de choisir entre les nettoyages pour lesquels la solution alcaline est utilisée en premier et ceux pour les- 


\section{TABLEAU 6}

Durée efficace et durée spécifique de nettoyage en fonction de l'ordre de passage des solutions détergentes

Effect of the cleaning procedure used on effective and specific times of cleaning

\begin{tabular}{|c|c|c|c|c|c|c|c|}
\hline & & \multicolumn{2}{|c|}{$\begin{array}{l}\text { Premier } \\
\text { nettoyage }\end{array}$} & \multicolumn{2}{|c|}{$\begin{array}{l}\text { Second } \\
\text { nettoyage }\end{array}$} & \multicolumn{2}{|c|}{$\begin{array}{l}\text { Durée } \\
\text { de nettoyage }\end{array}$} \\
\hline & & $t_{\mathrm{u}}, \mathrm{s}$ & $\frac{t_{u}}{M}, s^{-g} g^{-1}$ & $t_{u}, s$ & $\frac{t_{u}}{M}$, s.g $g^{-1}$ & $\begin{array}{c}\text { efficace, } \\
\mathrm{s}\end{array}$ & $\begin{array}{l}\text { spécifi- } \\
\text { que, } \\
\text { sg-1 }^{-1}\end{array}$ \\
\hline \multirow{5}{*}{ 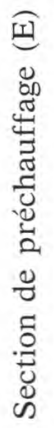 } & $\begin{array}{l}\text { Essai } 1 \\
\text { S-A }\end{array}$ & 540 & 8,2 & 120 & 10,2 & 660 & 18,4 \\
\hline & $\begin{array}{l}\text { Essai } 2 \\
\text { S-A }\end{array}$ & 242 & 4,8 & 238 & 16,9 & 480 & 21,7 \\
\hline & $\begin{array}{l}\text { Essai } 3 \\
\text { S-A }\end{array}$ & 174 & 5,2 & 58 & 4,8 & 232 & 10,0 \\
\hline & $\begin{array}{l}\text { Essai } 4 \\
\text { A-S }\end{array}$ & 242 & 5,0 & 162 & 2,6 & 404 & 7,6 \\
\hline & $\begin{array}{l}\text { Essai } 5 \\
\text { A-S }\end{array}$ & 174 & 5,3 & 138 & 3,9 & 312 & 9,1 \\
\hline \multirow{5}{*}{ 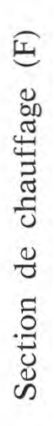 } & $\begin{array}{l}\text { Essai } 6 \\
\text { S-A }\end{array}$ & 820 & 32,8 & 202 & 1,1 & 1022 & 33,9 \\
\hline & $\begin{array}{l}\text { Essai } 7 \\
\text { S-A }\end{array}$ & 819 & 47,6 & 398 & 3,2 & 1217 & 50,8 \\
\hline & $\begin{array}{l}\text { Essai } 8 \\
\text { A-S }\end{array}$ & 400 & 6,1 & 270 & 56,2 & 670 & 62,3 \\
\hline & $\begin{array}{l}\text { Essai } 9 \\
\text { A-S }\end{array}$ & 200 & 1,5 & 460 & 52,9 & 660 & 54,4 \\
\hline & $\begin{array}{l}\text { Essai } 10 \\
\text { A-S }\end{array}$ & 650 & 5,0 & 560 & 42,5 & 1210 & 47,5 \\
\hline
\end{tabular}

$t_{u}$, temps utile de nettoyage ; $t_{u} / M$, temps spécifique de nettoyage ; $M$, masse totale enlevée par la solution considérée ; S-A, nettoyage alcalin suivi du nettoyage acide ; A-S, nettoyage acide suivi du nettoyage alcalin. $t_{u}$, effective time of cleaning; $t_{u} / M$, specific time of cleaning ; $M$, total mass removed by the considered solution; $S$ - $A$, alkaline cleaning followed by acid cleaning; $A-S$, acid cleaning followed by alkaline cleaning. 
quels elle est utilisée en second. Cependant le temps utile, lorsque le nettoyage alcalin est effectué en premier, est très important (supérieur à $800 \mathrm{~s}$ ). Ceci nous permet également de déconseiller les nettoyages utilisant l'hydroxyde de sodium comme première solution détergente sur cette zone dont le dépôt est essentiellement minéral.

\section{Discussion}

Hypothèses sur la structure des dépôts de la section de préchauffage $E$

Pour les nettoyages de type S-A, le passage de I'hydroxyde de sodium s'accompagne d'une diminution notoire du taux de colmatage jusqu'à une valeur très faible (environ $2 \%$ ) mais significativement différente de zéro. Bien que l'appareil ne soit pas tout à fait propre, la majeure partie du dépôt en volume a donc été enlevée. Or l'analyse des constituants nous a permis de montrer que toute la matière protéique et environ $50 \%$ de la matière minérale avaient été éliminées. La plus grande partie du dépôt en volume est donc formé par une couche constituée à la fois de protéines et de minéraux. S'il est logique de considérer que l'hydroxyde de sodium peut solubiliser les protéines, les minéraux sont par contre totalement insolubles en milieu basique. L'enlèvement de ces minéraux ne peut alors s'expliquer que par une structure de cette couche supérieure dans laquelle ils seraient emprisonnés dans la phase organique. Le nettoyage acide consécutif qui ramène le taux de colmatage à zéro élimine les minéraux restants soit $50 \%$ de la quantité initiale. Nous concluerons donc que ces minéraux formaient sans doute une couche peu épaisse mais dense située au contact de la paroi d'échange et sous la couche protéique et minérale qui était initialement en contact avec le lait.

Pour les nettoyages de type A-S, pendant le nettoyage acide le taux de colmatage ne change pratiquement pas par rapport à celui de l'appareil encrassé. Par contre la totalité des minéraux initialement présents a été enlevée. Ce résultat peut s'expliquer si nous admettons que l'acide a pénétré toute l'épaisseur du dépôt pour libérer les minéraux emprisonnés dans la couche supérieure et pour dissoudre la couche minérale compacte qui était en contact avec la plaque. L'hydroxyde de sodium utilisé ensuite ramène le taux de colmatage à zéro et élimine la totalité des protéines.

Le dépôt de la section de préchauffage serait donc composée de deux couches, une première minérale, compacte, en contact avec la surface des plaques et une seconde formant l'essentiel du volume très aérée et d'apparence très spongieuse, composée de protéines et de minéraux qui se développerait sur la couche compacte. Un pre- 
mier essai de photographie d'une coupe de dépôt effectuée au microscope électronique à balayage (photo 1) semble confirmer cette structure ; toutefois ces résultats demandent à être confirmés.

\section{Hypothèse sur la structure des dépôts de la section de chauffage F}

Pour les nettoyages de type S-A, l'étude de l'évolution des taux de colmatage indique que l'hydroxyde de sodium provoque un gonflement du dépôt et l'analyse des constituants éliminés montre qu'une forte partie des protéines $(80 \%)$ a été enlevée. Le nettoyage acide enlève les minéraux et le taux de colmatage diminue sans toutefois atteindre celui de l'appareil propre. Rappelons en effet que dans cette procédure le nettoyage est incomplet et qu'un gel protéique reste aux points de contact des plaques d'échange. Pour les nettoyages de type A-S, l'acide ne provoque aucune variation du taux de colmatage mais enlève tous les minéraux et un peu de protéines. La solution alcaline qui suit enlève le reste des protéines et ramène le taux de colmatage à celui de l'appareil propre.

A partir de ces résultats, beaucoup plus partiels que ceux du tronçon E, il est difficile d'établir des hypothèses approfondies sur la structure des dépôts du tronçon $\mathrm{F}$. Il semble néanmoins que la fraction minérale des dépôts, qui représente $70 \%$ à $80 \%$, constitue la base de leur structure.

\section{Conclusion}

Notre étude a porté essentiellement sur l'efficacité respective des solutions détergentes alcaline (hydroxyde de sodium) et acide (acide nitrique) utilisées lors du nettoyage des dépôts solides, formés pendant la stérilisation à Ultra Haute Température du lait par chauffage indirect au moyen d'échangeurs de chaleur à plaques. La température de nettoyage utilisée $\left(90^{\circ} \mathrm{C}\right)$ correspond industriellement au contexte des nettoyages finaux qui précèdent généralement un arrêt de la production. Les résultats obtenus sont donc à appliquer avec réserve au cas des nettoyages intermédiaires, qui mettent en œuvre des températures voisines de $140^{\circ} \mathrm{C}$. Celles-ci seront étudiées ultérieurement. Dans le cas de la section de préchauffage qui porte approximativement la température du lait de $80^{\circ} \mathrm{C}$ à $120^{\circ} \mathrm{C}$, le dépôt constitué d'environ $50 \%$ de matières organiques et de $40 \%$ de matières minérales est enlevé totalement par une procédure de nettoyage incluant l'utilisation de deux solutions, l'une alcaline, l'autre acide, quelque soit leur ordre de passage. Toutefois, la procédure pour laquelle la solution acide est utilisée en premier est significativement plus rapide. Dans le cas de la section de chauffage F qui porte le lait de $120^{\circ} \mathrm{C}$ à $140^{\circ} \mathrm{C}$, le dépôt est constitué d'environ $80 \%$ de matières minérales et de $10 \%$ de matières protéiques. Seule la procédure utilisant la solution acide en premier est totalement 
satisfaisante. En effet, dans l'autre cas, le premier nettoyage alcalin entraîne un gonflement irréversible du dépôt dont l'élimination par la solution acide s'avère alors très difficile. Lors du démontage de l'appareil nous observons un gel translucide aux points de contact des plaques, indiquant que cette procédure n'a pas permis un nettoyage complet. Il reste toutefois à vérifier si ce phénomène intervient aussi dans le cas des nettoyages intermédiaires à $140^{\circ} \mathrm{C}$.

Notre étude a également permis de montrer que la cinétique d'enlèvement des protéines par l'hydroxyde de sodium, et celle des minéraux par l'acide nitrique suivent le schéma réactionnel, composé des deux réactions en série proposé par Gallot-LAVALlée (1982). En rapport avec ce schéma, les hypothèses émises sur la structure et les mécanismes d'enlèvement des dépôts restent à confirmer.

D'un point de vue industriel, nous pouvons conseiller lors du nettoyage final des stérilisateurs à plaques U.H.T. de passer la solution acide avant celle d'hydroxyde de sodium. Cette procédure conduit à un nettoyage correct et plus rapide. Un nettoyage alcalin seul est à déconseiller. En effet, s'il agit efficacement dans le tronçon de préchauffage, il provoque un gonflement néfaste des dépôts sans les éliminer totalement dans le tronçon de chauffage. Un seul nettoyage acide a peu d'influence sur l'épaisseur du dépôt donc sur la perte de charge du tronçon considéré. Par ailleurs, sur la base de la méthode développée au paragraphe III 3.1., notre laboratoire a mis au point un capteur industriel d'encrassement indiquant à chaque instant le taux de colmatage. Son utilisation permet d'apprécier, sans démontage du stérilisateur, son degré de propreté. Un tel capteur pourrait permettre de mieux adapter les temps de nettoyage à la fois à la dimension du stérilisateur et aux impératifs technicoéconomiques imposés par les normes de propreté actuellement en vigueur.

Cependant, pour que notre étude sur le nettoyage des stérilisateurs à Ultra Haute Température soit complète, nous envisageons d'étudier désormais l'influence des paramètres qui pourraient agir sur l'efficacité du nettoyage telles que les températures, les vitesses de passage et les concentrations des solutions de nettoyage, la présence de tensio-actifs ou d'agents séquestrants, ainsi que l'intensité de l'encrassement au moment du nettoyage.

\section{Remerciements}

Ce travail a été réalisé grâce à une aide financière partielle de l'A.F.M.E. (Agence Française pour la Mấtrise de l'Energie) dans le cadre du Groupement V.E.R.E.D.I.A. (Valorisation Energétique des Résidus et Economies d'Energie dans les Industries Alimentaires) et du Conseil Régional du Nord-Pas-de-Calais. 


\section{Summary}

\section{Cleaning of milk UHT sterilizer : \\ Kinetics aspects with regard to acid and alkaline detergents}

One of the main disadvantages of indirect U.H.T. sterilization of milk is fouling of heat transfer surfaces. Energy consumption generally increases since pressure drops grow and heat transfer coefficients lower. Three times a day sterilizers need a cleaning in order to bring the exchange surface back to its original state. This procedure is expensive (immobilization time, thermal and mechanical energy consumptions, detergent costs...) and optimization of the cleaning process is only possible with a right knowledge of cleaning dynamics. The objective of this work is first to determine the effect of alkaline and acid cleaning according to the chemical composition of formed deposits (protein, fat and minerals) and secondly to evaluate the dynamic of the cleaning process.

Cold raw bulk milk was prepasteurized at $82.5^{\circ} \mathrm{C}$, then sterilized in a plate heat exchanger (total exchange area of $10 \mathrm{~m}^{2}$ ) at a flow rate of $400 \mathrm{l} / \mathrm{h}$. This sterilizer was comprised of a preheating section (E), a heating section ( $\mathrm{F})$ and a precooling section $(\mathrm{H})$. Milk was homogenized at $68^{\circ} \mathrm{C}$ during preheating and sterilized at $138^{\circ} \mathrm{C}$. At the end of each trial, the sterilizer was water rinsed and dismantled to weight the amount of deposit on each plate. One plate of each pass was kept in order to take samples for protein, fat and minerals analysis. The $\mathrm{E}$ and $\mathrm{F}$ sections were studied separately for cleaning. During the cleaning each plate used for sampling was replaced by a clean identical plate in order to keep the same configuration. Each section received two cleaning procedures. The first cleaning procedure (S-A) used first alkaline solution (sodium hydroxide $2 \%$ ) then acid solution (nitric acid $2 \%$ ), while the second cleaning procedure (A-S) used the same cleaning solutions but in reverse order. Cleaning temperature and flow rate were respectively $90^{\circ} \mathrm{C}$ and $400 \mathrm{l} / \mathrm{h}$. Cleaning kinetics were studied by sampling automatically cleaning solutions at the exit of the studied section to perform protein and minerals analysis. From these measurements cleaning rate were deduced. A differential pressure sensor was also used to determine on line the colmation level of the exchanger. By the assumption that the deposits of the two plates of each pass had the same composition, the total dry mass removed by the cleaning solutions was deduced and compared to the initial mass to be cleaned. From these results, it can be concluded that for E section, cleaning procedure (S-A) and cleaning procedure (A-S) led to correct cleaning, but cleaning is quicker for procedure (A-S). For F section, only cleaning procedure (A-S) is correct. During cleaning procedure (S-A) protein matter is not totally removed and when the apparatus is dismantled the heat exchange surface appeared still fouled in some parts. In all procedures, fat is supposed to be cleaned with protein. A study of cleaning rates was done. The apparent cleaning rate offered the same evolution whatever the cleaning procedure and whatever the matter to be cleaned. It was null at zero time, increased to a maximum, then decreased to zero. This trend is the same obtained by GALLOT-LAVALLÉE (1982) though our trials concerned an exchange surface 100 times more important on which the mass was not uniformly distributed. By fitting the two-steps Gallot-Lavallée model to our study, the velocity constants of each step were determined. In the preheating section $E$, during removal of protein by the alkaline solution the removing rate were significantly increased by using the cleaning procedure (A-S). On the other hand, during removal of minerals by the acid solution, each cleaning procedure acted differently on each step of the model. Efficient and specific cleaning times were defined to choose the best procedure for practical applications.

Key words : U.H.T. milk sterilizer - Alkaline and acid detergents - Kinetic Cleaning. 


\section{Références bibliographiques}

BURTon H., 1966. A comparaison between a hot-wire apparatus and a plate heat exchanger for determining the sensibility of milk to deposit formation. J. Dairy Res., 33, 317-324.

Cavell A.J., 1965. The colorimetric determination of phosphorus in plants materials. J. Sci. Food Agric., 6, 479-480.

Corrieu G., Lalande M., Ferret R., 1980. New monitoring equipment for the control and automation of milk pasteurization plants. In Food Process Engineering, 1, edited by Linko P., Malkki Y., Olkhu J., Larinkari J. J. Applied Science Publishers, London.

CoRrIeU G., 1981. State of the Art of cleaning surfaces. Eds. Hallstrom B., Lund D.B., Tragardh Ch., Tylosand, Sweden, April 6-9, 90-114.

Gallot-Lavallée T, 1982. Contribution à l'étude de la cinétique de nettoyage des pasteurisateurs de lait. Thèse de docteur ingénieur E.N.S.I.A., Massy.

Gallot-Lavallée T., Lalande M., Corrieu G., 1982. - An optical method to study the kinetics of cleaning milk deposits by sodium hydroxyde. J. Food Process Eng., 5, 131-143,

Gallot-Lavallée T., Lalande M., Corrieu G., 1984. Cleaning kinetics modeling of holding tubes fouled during milk pasteurization. J. Food Process Eng., 7, 123-142.

Gornall A.G., BaRdawill C., David N.M., 1949. Détermination des protéines par la méthode du Biuret. I. Biol. Chem., 177, 751-752.

Helaine E., 1977. Dosage d'ammoniaque dans le lait par électrode spécifique, Ind. Aliment. Agric., 6, 581-588.

Iтo R., Sato M., SuzukI M., 1967. Formation of milk deposits on heat exchange surfaces in U.H.T. pasteurizing plants, Dairy Sci., Abstr., 4092-4094.

Jennings W.G., 1957. Circulation cleaning. J. Dairy Sci., 40, 1471-1479.

Jennings W.G., 1959 a. Circulation cleaning. II. Effects of entrained air. J. Dairy Sci., 42, 476-479.

Jennings W.G., 1959 b. Circulation cleaning. III. The kinetics of a simple detergent system. I. Dairy Sci., 42, 1763-1771.

JENNINGS W.G., 1963. An interpretive review of detergency for the food Technologist. Food Technol., 17, 7, 53-59.

Kulkarni S.M., Maxcy R.B., ARnold R.G., 1975. Evaluation of soil deposition and removal processes : an interpretative review. J. Dairy Sci., 58, 12, 1922-1936.

lalande M., Corrieu G., Daufin G., Gallot-Lavallée T., 1981. Etude des cinétiques d'encrassement et de nettoyage d'un pasteurisateur de lait à plaques. Contrat D.G.R.S.T. $n^{\circ} 7970375$.

Lalande M., Corrieu G., 1979. Brevet 1979/09.969.

LALANDE M., Tissier J.P., CoRrieu G., 1984. Fouling of a plate heat exchanger used in milk Ultra High Temperature sterilization. J. Dairy Res., 51, 557-568.

LySTER R.L.J., 1965. The composition of milk deposits in a Ultra High Temperature plant. J. Dairy Res., 32, 203-208.

SCHLUSSLER H.J., 1970. Zur Reinigung fester Oberflächer in der Lebensmittelindustrie. Milchwissenschaft, 25, 3, 133-149.

SCHLUSSLER H.J., 1976. Zur Kinetic von Reinigungworgängen an fester Oberflächen. Brauwissenschaft, 29, 9, 263-268.

Тном R., 1970. - Uber die Struktier des Milchansatzen in Plattenerhitzen. Milchwissenshaft, 25, 519-528. 